Review Article

\title{
Zingiberis Rhizoma Recens: A Review of Its Traditional Uses, Phytochemistry, Pharmacology, and Toxicology
}

\author{
Xing Li, Mingyue Ao, Chunling Zhang, Shunming Fan, Zhimin Chen, and Lingying Yu $(10$ \\ College of Pharmacy, Chengdu University of Traditional Chinese Medicine, Chengdu 6111137, China \\ Correspondence should be addressed to Lingying Yu; yly111666@163.com
}

Received 22 October 2020; Revised 25 January 2021; Accepted 10 February 2021; Published 2 March 2021

Academic Editor: Armando Zarrelli

Copyright (c 2021 Xing Li et al. This is an open access article distributed under the Creative Commons Attribution License, which permits unrestricted use, distribution, and reproduction in any medium, provided the original work is properly cited.

\begin{abstract}
Zingiberis Rhizoma Recens (ZRR, the fresh rhizoma of Zingiber officinale Roscoe) is a widely used traditional Chinese medicine (TCM). It is also a traditional spice, widely used around the world. The present paper reviews advances in research relating to the botany, ethnopharmacology, phytochemistry, pharmacology, and toxicology of Zingiberis Rhizoma Recens. In addition, this review also discusses some significant issues and the potential direction of future research on Zingiberis Rhizoma Recens. More than 100 chemical compounds have been isolated from Zingiberis Rhizoma Recens, including gingerols, essential oils, diarylheptanoids, and other compounds. Modern studies have confirmed that Zingiberis Rhizoma Recens has pharmacological effects on the nervous system and cardiovascular and cerebrovascular systems, as well as antiemetic, antibacterial, antitumor, antiinflammatory, and antioxidant effects. However, the modern studies of Zingiberis Rhizoma Recens are still not complete and more bioactive components and potential pharmacological effects need to be explored in the future. There is no unified standard to evaluate the quality and clinical efficacy of Zingiberis Rhizoma Recens. Therefore, we should establish reasonable, accurate, and reliable quality control standards to make better use of Zingiberis Rhizoma Recens.
\end{abstract}

\section{Introduction}

Zingiberis Rhizoma Recens (ZRR) is the fresh rhizoma of Zingiber officinale Roscoe and has a long history as an effective traditional Chinese medicine. The use of ZRR can be traced from the Shen Nong Ben Cao Jing. In Chinese Pharmacopoeia, ZRR has the function of relieving exterior syndrome and dispersing cold and warming middle energizer to arrest vomiting. It is mostly used to treat cold, vomiting, and cough caused by wind weather. ZRR is distributed in China, India, Indonesia, Nigeria, and other countries and is famous for its antiemetic effect.

In recent years, many researchers have carried out indepth research on the phytochemical and pharmacological effects of ZRR and made significant progress. Modern phytochemical studies have shown that the main components of ZRR include gingerols, essential oils, diarylheptanoids, and other compounds. Pharmacological studies have also shown that ZRR has a wide range of pharmacological effects, including effects on the nervous system and cardiovascular and cerebrovascular systems, as well as antiemetic, antibacterial, antitumor, anti-inflammatory, and antioxidant effects. This review provides information on the botanical characterizations, traditional usages, chemical constituents, pharmacological activities, toxicity, and quality control of ZRR. In this paper, we hope to have a comprehensive understanding of the effects of ZRR and also provide a basis for further research.

\section{Botany}

ZRR is known as "Shengjiang" in China and is the fresh rhizoma of Zingiber officinale Roscoe (Z. officinale) (Figure 1), and the plant is widely cultivated in central, southeast, and southwest of China.

As far as the growing environment is concerned, $Z$. officinale is generally distributed in the tropical and subtropical regions and is widely cultivated in many countries of southern and eastern of Asia including India, Indonesia, Nigeria, and Thailand.

Z. officinale, a member of the Zingiberaceae family, likes a warm and humid climate. Its cold resistance and drought resistance are weak, and its plants can only grow in the frost- 


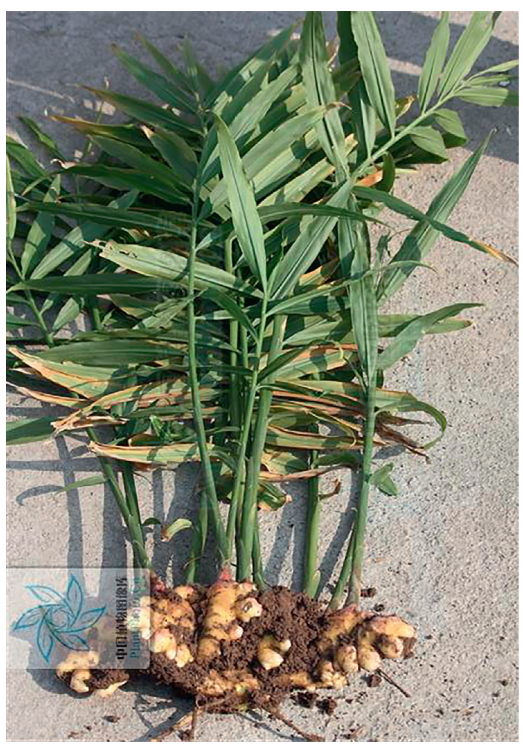

(a)

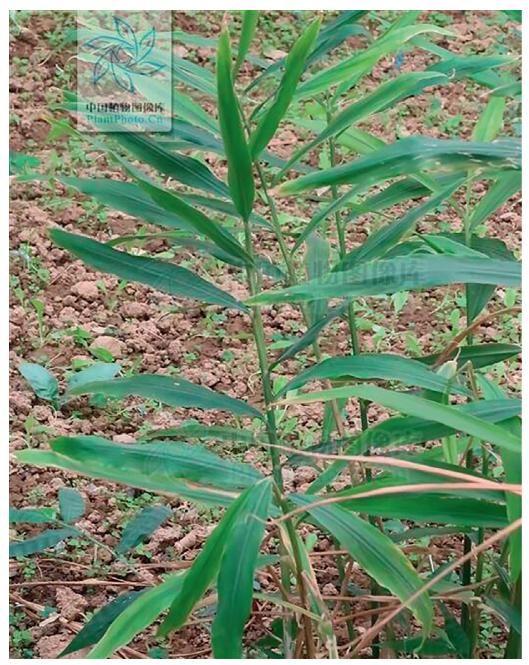

(c)

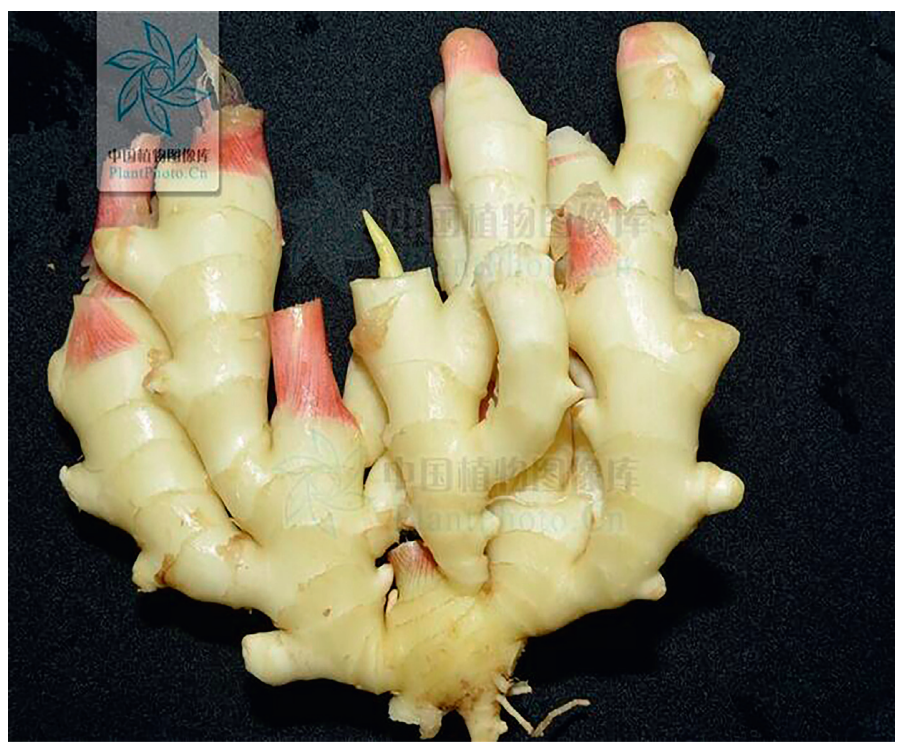

(b)

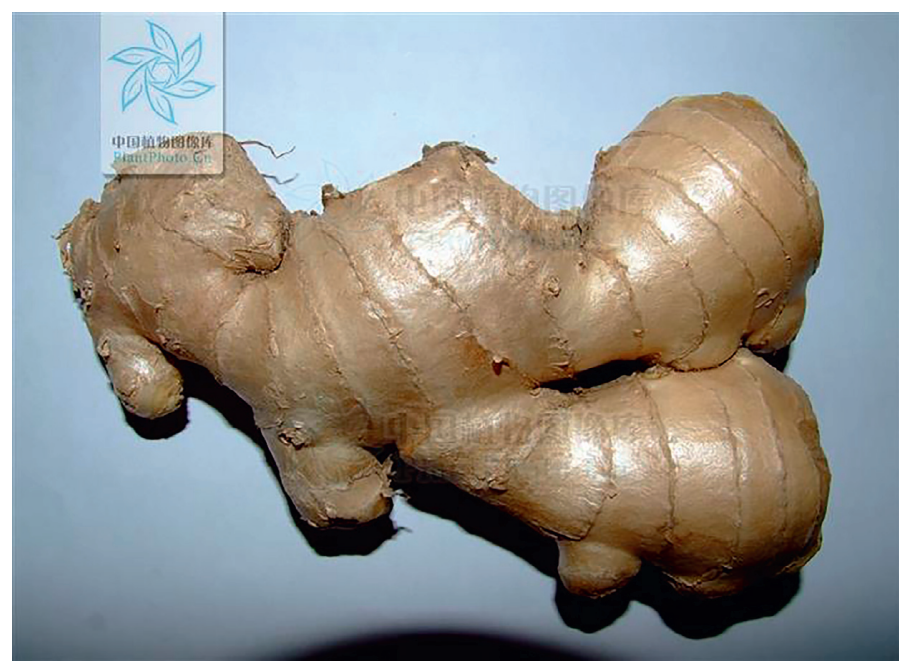

(d)

Figure 1: The entire Z. officinale plant (a), the underground part of Z. officinale (b), the aboveground part of Z. officinale (c), and ZRR (the fresh rhizoma of $Z$. officinale) (d) (cited from Flora Republicae Populairs Sinicae at http://ppbc.iplant.cn).

free period. The optimum temperature for growth is $25-28^{\circ} \mathrm{C}$. It primarily thrives in moist and nonwater sandy loam that is loose and fertile. The plant is approximately $0.5-1 \mathrm{~m}$ tall and has fleshy, branched rhizomes. This plant is aromatic and spicy. The leaves are lanceolate or linear-lanceolate, its apex is acuminate, and its base is attenuated to narrow, glabrous, and sessile. The inflorescence is ovoid, the bracts are ovate, pale green, or yellowish at margin, and its apex is mucronate. The calyx tube is about $1 \mathrm{~cm}$ long; the flower is yellowish green, and the lobes are lanceolate. The flowering period is autumn [1]. ZRR is harvested in autumn and winter, the fibrous roots are removed, and then rhizoma is used for medicinal purposes.

\section{Ethnopharmacology}

3.1. Traditional Use. ZRR is a kind of Chinese herbal medicine with a wide range of pharmacological effects and plays an indispensable role in the healthcare industry in China for a long time.

In the Chinese Pharmacopoeia (version 2020), this herb is described as being slight warm in property and acrid in flavour. It affects the spleen, stomach, and lung and acts to relieve exterior syndrome and disperse cold, warm middle energizer to arrest vomiting, dissipate phlegm and relieve cough, and resolve the toxin of fish and crabs; therefore, it can be applied in the treatment of common cold due to wind-cold, vomiting due to stomach cold, cough resulting from cold phlegm, and poison from fish and crabs. The recommended dosage is $3-10 \mathrm{~g}$. Because ZRR has a strong effect on promoting fire in the body and may cause damage to Yang Qi, individuals with excessive heat or yin deficiency and internal heat should avoid to use it [2].

In China, the use of ZRR can be traced from the spring and autumn periods (770-476 BC), or even earlier. It was 
mentioned in the Shen Nong Ben Cao Jing and was classified as a middle product. Middle product usually refers to nontoxic or very small toxicity, which has the effect of tonifying deficiency and strengthening weakness or eliminating pathogenic factors and resisting diseases. It is mainly used to adjust the body, but it cannot be taken for a long time [3].

ZRR has a wide range of effects. It is often used in combination with other Chinese medicines but is generally not used alone. In the major prescription books in ancient China, Shang Han Za Bing Lun, there are 71 prescriptions containing ZRR, including 38 in the Treatise on Shang Han Lun and 33 in the Outline of Jin Gui Yao Lue. The parts of formulations are shown in Table 1.

3.2. Processing. Before Chinese herbal medicines are used, they are usually processed by the methods of cleansing, cutting, heating, or combining with processed excipients. The purpose of these processes is to improve the efficacy or reduce the toxicity of the product.

Jin Gui Yao Lue recorded the method of cutting in the Han dynasty. According to Lei Gong Pao Zhi Lun, there was a method of peeling in the Northern and Southern dynasties. Lishang Xuduan Fang recorded that there was the method of roasting in the Tang dynasty. The Ming dynasty had the stirfry (Jing Yue Quan Shu), the sun-drying method (Ben Cao Meng Quan), and so on. In the current version of the Chinese Pharmacopoeia, only two processing methods have been reported: sun-drying or low-temperature drying.

However, processing has an unavoidable impact on the composition and efficacy of medicinal materials. We also reviewed the content change studies of ZRR before and after processing and found that the content of volatile oil decreased and the content of gingerols and polysaccharides increased [6-8]. The changes of these components are mainly quantitative, and the deep-seated relationship between them and the changes of pharmacodynamics require further study. Therefore, different processed products have different clinical effects and appropriate processed products should be selected according to the condition.

\section{Phytochemistry}

A literature search identified over 100 chemical constituents that have been isolated from $Z$. officinale. The main constituents are gingerols, essential oils, diarylheptanoids, and others. The compounds isolated are listed in Tables 2-4, and their corresponding structures are also presented.

4.1. Gingerols. Gingerol is the general term of the pungent ingredients in $Z$. officinale, which is a mixture of many substances. Gingerols are not only the main components of characteristic spicy flavor, but also the main functional components of various biological activities. The structure of gingerols contains the 3-methoxy-4-hydroxyphenyl functional group. According to the different fatty chains connected to this functional group, it can be divided into the following types:
4.1.1. Gingerols. At present, there are more than ten clear gingerols, including 6-, 8-, 10-, and 12-gingerol. The properties and structures of these components are similar. There are $C_{3}$ carbonyl and $C_{5}$ hydroxy (i.e., $\beta$-hydroxyketone structure) in their molecular structures, which makes the chemical properties of gingerols extremely unstable. For example, in acid condition $(\mathrm{pH}<4.5)$, the active hydrogen of $\mathrm{C}_{4}$ is easy to dehydrate with the hydroxyl of $\mathrm{C}_{5}$ to form shogaols; in heating or alkaline condition, the carboncarbon bond between $\mathrm{C}_{4}$ and $\mathrm{C}_{5}$ breaks to form zingerone and corresponding aldehydes. The transformation of gingerols under different conditions is shown in Figure 2. In addition, the length of the side chain, the acetoxy group at the 3, 5 position, the methoxy group on the aromatic ring, and the phenolic hydroxyl functional group all affect the biological activity of gingerols [11].

4.1.2. Shogaols. It is considered that the content of shogaols in Z. officinale is very small, which is formed by dehydration of gingerols during storage and processing. The content of shogaols in $Z$. officinale is 6-shogaol $>10$-shogaol $>8$ shogaol > 4-shogaol [21].

4.1.3. Others. In addition, gingerols also contain other components, such as zingerone, paradols, gingerdiones, and gingerdiols. The part of the structural formula is shown in Figure 3.

4.2. Essential Oils. Essential oil is also one of the main components of $Z$. officinale and is mainly composed of pinene, phellandrene, and camphene [22]. The total content of essential oil is $0.25 \%-3.0 \%$. The essential oil component is dominated by terpenoids and is mainly composed of sesquiterpene carbohydrate $(50 \%-66 \%)$ and sesquiterpene oxide $(17 \%)$. The part of structural formula is shown in Figure 4.

4.3. Diarylheptanoids. Diarylheptanoids are a group of substances with nuclear structure of 1, 7-diarylheptanoid. According to their structural characteristics, they can be divided into two groups: cyclic diarylheptanoid and linear diarylheptanoid. The chemical structure of linear diarylheptanoid is characterized by the position of two phenyl substituents at 1, 7 of heptane skeleton, the substituents on phenyl are methoxy or hydroxy, and the substituents are in para- or interposition; there are 3 types of substituted diphenyl groups: phenyl, 4-hydroxyphenyl, and 3-methoxy4-hydroxyphenyl [23]. The structural formula is shown in Figure 5.

4.4. Other Components. Besides the aforementioned, phenylpropanoids, flavonoids, nucleosides, polysaccharides, proteins, cellulose, and a large number of carbohydrates and trace elements have been isolated from $Z$. officinale [9]. Zhang [24] found that it contained mineral elements 
TABLE 1: Traditional and clinical uses of ZRR in China.

\begin{tabular}{|c|c|c|c|c|}
\hline Preparation name & Main compositions & Formulation & Traditional and clinical uses & Ref \\
\hline $\begin{array}{l}\text { Shengjiang Xiexin } \\
\text { Tang }\end{array}$ & $\begin{array}{c}\text { Zingiberis Rhizoma Recens, Glycyrrhizae } \\
\text { Radix Et Rhizoma Praeparata Cum Melle, } \\
\text { Ginseng Radix Et Rhizoma, Zingiberis } \\
\text { Rhizoma, Scutellariae Radix, Pinelliae } \\
\text { Rhizoma, Coptidis Rhizoma, Jujubae } \\
\text { Fructus. }\end{array}$ & Decoction & $\begin{array}{l}\text { Epigastralgia, gastroptosis, gastrectasis, chronic } \\
\text { gastritis, and other diseases. }\end{array}$ & [4] \\
\hline $\begin{array}{l}\text { Guizhi Shengjiang } \\
\text { Zhishi Tang }\end{array}$ & $\begin{array}{l}\text { Cinnamomi Ramulus, Zingiberis Rhizoma } \\
\text { Recens, Aurantii Fructus Immaturus. }\end{array}$ & Decoction & $\begin{array}{l}\text { Chronic gastritis, gastroptosis, or coronary heart } \\
\text { disease and angina pectoris caused by chest } \\
\text { stuffiness and cardialgia, phlegm retention, and } \\
\text { deficiency of Yang Qi in the heart and stomach. }\end{array}$ & {$[5]$} \\
\hline Guizhi Tang & $\begin{array}{c}\text { Cinnamomi Ramulus, Paeoniae Radix Alba, } \\
\text { Zingiberis Rhizoma Recens, Jujubae Fructus, } \\
\text { Glycyrrhizae Radix Et Rhizoma Praeparata } \\
\text { Cum Melle }\end{array}$ & Decoction & $\begin{array}{l}\text { Common cold, influenza, unexplained low fever, } \\
\text { and deficiency syndrome of exogenous wind and } \\
\text { cold. }\end{array}$ & {$[4]$} \\
\hline $\begin{array}{l}\text { Houpo Shengjiang } \\
\text { Gancao Banxia } \\
\text { Renshen Tang }\end{array}$ & $\begin{array}{l}\text { Magnoliae Officinalis Cortex, Zingiberis } \\
\text { Rhizoma Recens, Pinelliae Rhizoma, } \\
\text { Glycyrrhizae Radix Et Rhizoma Praeparata } \\
\text { Cum Melle, Ginseng Radix Et Rhizoma. }\end{array}$ & Decoction & Gastric ulcer and abdominal distension. & {$[4]$} \\
\hline Wuzhuyu Tang & $\begin{array}{c}\text { Euodiae Fructus, Zingiberis Rhizoma } \\
\text { Recens, Ginseng Radix Et Rhizoma, Jujubae } \\
\text { Fructus. }\end{array}$ & Decoction & $\begin{array}{c}\text { Chronic gastritis, vomiting during pregnancy, } \\
\text { neurogenic vomiting, nervous headache, and } \\
\text { aural vertigo. }\end{array}$ & {$[4]$} \\
\hline Zhen Wu Tang & $\begin{array}{l}\text { Poria, Paeoniae Radix Alba, Zingiberis } \\
\text { Rhizoma Recens, Typhonii Rhizoma, } \\
\text { Atractylodis Macrocephalae Rhizoma. }\end{array}$ & Decoction & $\begin{array}{l}\text { Chronic glomerulonephritis, cardiogenic } \\
\text { edema, hypothyroidism, chronic bronchitis, } \\
\text { chronic enteritis, and intestinal tuberculosis. }\end{array}$ & {$[4]$} \\
\hline
\end{tabular}

(including $\mathrm{Fe}, \mathrm{Al}, \mathrm{Mn}, \mathrm{Na}$, and $\mathrm{Zn}$ ) and 17 amino acids, such as Asp, Glu, Arg, Leu, and Gly.

\section{Pharmacological Activities}

In recent years, many researchers have conducted in-depth research on the pharmacological effects of extracts or isolated compounds from ZRR and have found that ZRR has a wide range of pharmacological effects (Table 5). Modern pharmacological studies confirmed the traditional efficacy of ZRR, particularly in antiemetic. The main mechanism of action may be related to the 5-HT receptor, but further studies are required to define the related mechanisms. Pharmacological studies in animals experiments also showed that ZRR has potential as a treatment for Alzheimer's disease (AD) and cognition. The antioxidant and anti-inflammatory effects of $\mathrm{ZE}$ and its isolated compounds also indicated pharmacological effects in cardiovascular and cerebrovascular fields. In addition, in vitro and in vivo pharmacological studies have shown that ZE and compounds have antibacterial and antitumor effects.

We will introduce the research progress of modern pharmacology of ZRR from its effects on the nervous system and cardiovascular and cerebrovascular systems and will discuss reported antiemetic, antibacterial, antitumour, antiinflammatory, and antioxidative effects.

5.1. Pharmacological Effects on the Nervous System. Modern pharmacological studies have shown that ZRR can be used to treat nervous system diseases, such as $\mathrm{AD}$, and has the effect of improving cognitive function. Although the mechanisms of these effects have been studied, to better develop the medicinal value of $Z R R$, the related mechanisms of action require more study.

5.1.1. Treatment of Alzheimer's Disease. Experimental evidence suggested that learning and memory impairment in mouse could be improved by 6 -shogaol both in vitro and in vivo. 6-Shogaol decreased the levels of CysLT1R/cathepsin B and amyloid-beta in the brain of mice. Therefore, 6-shogaol may be beneficial for treating $\mathrm{AD}$ due to its role as a CysLT1R and cathepsin B inhibitor [34]. Further research showed that 6-gingerol activated Akt activity and inhibited GSK-3 $\beta$ activity, thereby protecting PC12 cells against $A \beta_{1-42}$-induced apoptosis through the PI3K/Akt/GSK-3 $\beta$ signaling pathway, suggesting that 6-gingerol may be one of the effective intervention measures for $\mathrm{AD}$ [35].

Another researcher investigated whether fermented ginger (FG) had a neuroprotective effect in an AD mouse model. These researchers found that FG alleviated memory dysfunction induced by the $A \beta_{1-42}$ plaque toxicity and inhibited $A \beta_{1-42}$ plaque-induced neuronal cell loss in the CA 3 region of the hippocampus. Thus, these studies showed the antiamnesic effects of FG against the $A \beta_{1-42}$ plaque toxicity by inhibiting neuronal cell loss and synaptic disturbance, besides suggesting that FG can alleviate AD-like memory dysfunction and neuronal degradation [36].

5.1.2. Improving Cognition. Research using an improved Morris water maze test revealed that ZRR $(4 \mathrm{~g} / \mathrm{kg})$ reduced the impairment of lead-induced learning and memory in rats [37]. Zhang et al. studied whether 6-gingerol could reduce memory dysfunction and neuroinflammation in rats by administration of lipopolysaccharide (LPS). The 
TABLE 2: Gingerols isolated from Z. officinale.

\begin{tabular}{|c|c|c|c|c|}
\hline No. & & Chemical component & Formula & Ref. \\
\hline \multicolumn{5}{|c|}{ Gingerols } \\
\hline 1 & & 4-Gingerol & $\mathrm{C}_{15} \mathrm{H}_{22} \mathrm{O}_{4}$ & [9] \\
\hline 2 & & Methyl-4-gingerol & $\mathrm{C}_{16} \mathrm{H}_{24} \mathrm{O}_{4}$ & [9] \\
\hline 3 & & 5-Gingerol & $\mathrm{C}_{16} \mathrm{H}_{24} \mathrm{O}_{4}$ & [9] \\
\hline 4 & & 6-Gingerol & $\mathrm{C}_{17} \mathrm{H}_{26} \mathrm{O}_{4}$ & {$[10]$} \\
\hline 5 & & Methyl-6-gingerol & $\mathrm{C}_{18} \mathrm{H}_{28} \mathrm{O}_{4}$ & {$[10]$} \\
\hline 6 & & 8-Gingerol & $\mathrm{C}_{19} \mathrm{H}_{30} \mathrm{O}_{4}$ & {$[10]$} \\
\hline 7 & & 10-Gingerol & $\mathrm{C}_{21} \mathrm{H}_{34} \mathrm{O}_{4}$ & {$[10]$} \\
\hline 8 & & 12-Gingerol & $\mathrm{C}_{23} \mathrm{H}_{38} \mathrm{O}_{4}$ & {$[11]$} \\
\hline 9 & & Acetoxy-4-gingerol & $\mathrm{C}_{17} \mathrm{H}_{24} \mathrm{O}_{5}$ & {$[12]$} \\
\hline 10 & & Acetoxy-6-gingerol & $\mathrm{C}_{19} \mathrm{H}_{28} \mathrm{O}_{5}$ & {$[12]$} \\
\hline 11 & & Acetoxy-8-gingerol & $\mathrm{C}_{21} \mathrm{H}_{32} \mathrm{O}_{5}$ & {$[10]$} \\
\hline 12 & & Acetoxy-10-gingerol & $\mathrm{C}_{23} \mathrm{H}_{36} \mathrm{O}_{5}$ & {$[10]$} \\
\hline \multicolumn{5}{|c|}{ Shogaols } \\
\hline 13 & & 4-Shogaol & $\mathrm{C}_{15} \mathrm{H}_{20} \mathrm{O}_{3}$ & {$[10]$} \\
\hline 14 & & Methyl-4-shogaol & $\mathrm{C}_{16} \mathrm{H}_{22} \mathrm{O}_{3}$ & [9] \\
\hline 15 & & 5-Shogaol & $\mathrm{C}_{16} \mathrm{H}_{22} \mathrm{O}_{3}$ & [9] \\
\hline 16 & & 6-Shogaol & $\mathrm{C}_{17} \mathrm{H}_{24} \mathrm{O}_{3}$ & {$[10]$} \\
\hline 17 & & Methyl-6-shogaol & $\mathrm{C}_{18} \mathrm{H}_{26} \mathrm{O}_{3}$ & [9] \\
\hline 18 & & 8-Shogaol & $\mathrm{C}_{19} \mathrm{H}_{28} \mathrm{O}_{3}$ & {$[10]$} \\
\hline 19 & & Methyl-8-shogaol & $\mathrm{C}_{20} \mathrm{H}_{30} \mathrm{O}_{3}$ & [9] \\
\hline 20 & & 10-Shogaol & $\mathrm{C}_{21} \mathrm{H}_{32} \mathrm{O}_{3}$ & {$[10]$} \\
\hline 21 & & 12-Shogaol & $\mathrm{C}_{23} \mathrm{H}_{36} \mathrm{O}_{3}$ & {$[10]$} \\
\hline \multicolumn{5}{|c|}{ Paradols } \\
\hline 22 & & 6-Paradol & $\mathrm{C}_{17} \mathrm{H}_{26} \mathrm{O}_{3}$ & {$[10]$} \\
\hline 23 & & Methyl-6-paradol & $\mathrm{C}_{18} \mathrm{H}_{28} \mathrm{O}_{3}$ & [9] \\
\hline 24 & & 8-Paradol & $\mathrm{C}_{19} \mathrm{H}_{30} \mathrm{O}_{3}$ & {$[13]$} \\
\hline 25 & & Methyl-8-paradol & $\mathrm{C}_{20} \mathrm{H}_{32} \mathrm{O}_{3}$ & [9] \\
\hline 26 & & 10-Paradol & $\mathrm{C}_{21} \mathrm{H}_{34} \mathrm{O}_{3}$ & {$[12]$} \\
\hline 27 & Zingerone & & $\mathrm{C}_{11} \mathrm{H}_{14} \mathrm{O}_{3}$ & {$[13]$} \\
\hline \multicolumn{5}{|c|}{ Gingerdione } \\
\hline 28 & & 6-Gingerdione & $\mathrm{C}_{17} \mathrm{H}_{24} \mathrm{O}_{4}$ & {$[12]$} \\
\hline 29 & & 8-Gingerdione & $\mathrm{C}_{19} \mathrm{H}_{28} \mathrm{O}_{4}$ & [13] \\
\hline 30 & & 10-Gingerdione & $\mathrm{C}_{21} \mathrm{H}_{32} \mathrm{O}_{4}$ & {$[10]$} \\
\hline 31 & & 12-Gingerdione & $\mathrm{C}_{23} \mathrm{H}_{36} \mathrm{O}_{4}$ & [9] \\
\hline 32 & & 1-Dehydro-6-gingerdione & $\mathrm{C}_{17} \mathrm{H}_{22} \mathrm{O}_{4}$ & {$[13]$} \\
\hline 33 & & 1-Dehydro-8-gingerdione & $\mathrm{C}_{19} \mathrm{H}_{26} \mathrm{O}_{4}$ & {$[13]$} \\
\hline 34 & & 1-Dehydro-10-gingerdione & $\mathrm{C}_{21} \mathrm{H}_{30} \mathrm{O}_{4}$ & {$[13]$} \\
\hline 35 & & 1-Dehydro-12-gingerdione & $\mathrm{C}_{23} \mathrm{H}_{34} \mathrm{O}_{4}$ & {$[13]$} \\
\hline \multicolumn{5}{|c|}{ Gingerdiol } \\
\hline 36 & & 4-Gingerdiol & $\mathrm{C}_{15} \mathrm{H}_{24} \mathrm{O}_{4}$ & [9] \\
\hline 37 & & 6-Gingerdiol & $\mathrm{C}_{17} \mathrm{H}_{28} \mathrm{O}_{4}$ & {$[10]$} \\
\hline 38 & & 8-Gingerdiol & $\mathrm{C}_{19} \mathrm{H}_{32} \mathrm{O}_{4}$ & [9] \\
\hline 39 & & 10-Gingerdiol & $\mathrm{C}_{21} \mathrm{H}_{36} \mathrm{O}_{4}$ & [9] \\
\hline 40 & & 5-Acetoxy-4-gingerdiol & $\mathrm{C}_{17} \mathrm{H}_{26} \mathrm{O}_{5}$ & [9] \\
\hline 41 & & $\begin{array}{l}\text { Methyl-5-acetoxy-4- } \\
\text { gingerdiol }\end{array}$ & $\mathrm{C}_{18} \mathrm{H}_{28} \mathrm{O}_{5}$ & [9] \\
\hline 42 & & 5-Acetoxy-6-gingerdiol & $\mathrm{C}_{19} \mathrm{H}_{30} \mathrm{O}_{5}$ & {$[10]$} \\
\hline 43 & & $\begin{array}{l}\text { Methyl-5-acetoxy-6- } \\
\text { gingerdiol }\end{array}$ & $\mathrm{C}_{20} \mathrm{H}_{32} \mathrm{O}_{5}$ & {$[10]$} \\
\hline 44 & & Diacetoxy-4-gingerdiol & $\mathrm{C}_{19} \mathrm{H}_{28} \mathrm{O}_{6}$ & {$[10]$} \\
\hline 45 & & $\begin{array}{l}\text { Methyl-diacetoxy-4- } \\
\text { gingerdiol }\end{array}$ & $\mathrm{C}_{20} \mathrm{H}_{30} \mathrm{O}_{6}$ & {$[9]$} \\
\hline 46 & & Diacetoxy-6-gingerdiol & $\mathrm{C}_{21} \mathrm{H}_{32} \mathrm{O}_{6}$ & {$[12]$} \\
\hline 47 & & $\begin{array}{l}\text { Methyl-diacetoxy-6- } \\
\text { gingerdiol }\end{array}$ & $\mathrm{C}_{22} \mathrm{H}_{34} \mathrm{O}_{6}$ & {$[12]$} \\
\hline 48 & & Diacetoxy-8-gingerdiol & $\mathrm{C}_{23} \mathrm{H}_{36} \mathrm{O}_{6}$ & [9] \\
\hline 49 & & $\begin{array}{l}\text { Methyl-diacetoxy-8- } \\
\text { gingerdiol }\end{array}$ & $\mathrm{C}_{24} \mathrm{H}_{38} \mathrm{O}_{6}$ & [9] \\
\hline
\end{tabular}

TABle 3: Essential oils isolated from $Z$. officinale.

\begin{tabular}{|c|c|c|c|}
\hline No. & Chemical component & Formula & Ref. \\
\hline \multicolumn{4}{|c|}{ Monoterpenoids } \\
\hline 50 & $\alpha$-Pinene & $\mathrm{C}_{10} \mathrm{H}_{16}$ & [14] \\
\hline 51 & Camphene & $\mathrm{C}_{10} \mathrm{H}_{16}$ & [14] \\
\hline 52 & $\beta$-Myrcene & $\mathrm{C}_{10} \mathrm{H}_{16}$ & [15] \\
\hline 53 & $\beta$-Phellandrene & $\mathrm{C}_{15} \mathrm{H}_{16}$ & [14] \\
\hline 54 & 1, 8-Cineole & $\mathrm{C}_{10} \mathrm{H}_{18} \mathrm{O}$ & [14] \\
\hline 55 & p-Cymene & $\mathrm{C}_{10} \mathrm{H}_{14}$ & [15] \\
\hline 56 & $\alpha$-Terpinene & $\mathrm{C}_{10} \mathrm{H}_{16}$ & [16] \\
\hline 57 & Geraniol & $\mathrm{C}_{10} \mathrm{H}_{18} \mathrm{O}$ & [14] \\
\hline 58 & $\alpha$-Terpineol & $\mathrm{C}_{10} \mathrm{H}_{18} \mathrm{O}$ & [14] \\
\hline 59 & Tricyclene & $\mathrm{C}_{10} \mathrm{H}_{16}$ & {$[14]$} \\
\hline 60 & $\beta$-Pinene & $\mathrm{C}_{10} \mathrm{H}_{16}$ & [14] \\
\hline 61 & $\alpha$-Phellandrene & $\mathrm{C}_{15} \mathrm{H}_{16}$ & [14] \\
\hline 62 & Sabinene & $\mathrm{C}_{10} \mathrm{H}_{16}$ & [14] \\
\hline 63 & Citronellal & $\mathrm{C}_{10} \mathrm{H}_{18} \mathrm{O}$ & [14] \\
\hline 64 & Terpinolene & $\mathrm{C}_{10} \mathrm{H}_{16}$ & [14] \\
\hline 65 & Bornyl acetate & $\mathrm{C}_{12} \mathrm{H}_{20} \mathrm{O}_{2}$ & [15] \\
\hline 66 & Nerol & $\mathrm{C}_{10} \mathrm{H}_{18} \mathrm{O}$ & [16] \\
\hline 67 & Linalool & $\mathrm{C}_{10} \mathrm{H}_{18} \mathrm{O}$ & [14] \\
\hline 68 & Camphor & $\mathrm{C}_{10} \mathrm{H}_{16} \mathrm{O}$ & [15] \\
\hline 69 & Borneol & $\mathrm{C}_{10} \mathrm{H}_{18} \mathrm{O}$ & [14] \\
\hline 70 & Citronellol & $\mathrm{C}_{10} \mathrm{H}_{20} \mathrm{O}$ & [14] \\
\hline 71 & Geranial & $\mathrm{C}_{10} \mathrm{H}_{16} \mathrm{O}$ & [17] \\
\hline 72 & Geranic acid & $\mathrm{C}_{10} \mathrm{H}_{16} \mathrm{O}_{2}$ & {$[17]$} \\
\hline 73 & Citronellyl acetate & $\mathrm{C}_{12} \mathrm{H}_{22} \mathrm{O}_{2}$ & [14] \\
\hline 74 & Geranyl acetate & $\mathrm{C}_{12} \mathrm{H}_{20} \mathrm{O}_{2}$ & [14] \\
\hline \multicolumn{4}{|c|}{ Sesquiterpenoids } \\
\hline 75 & $\alpha$-Zingiberene & $\mathrm{C}_{15} \mathrm{H}_{24}$ & [14] \\
\hline 76 & $\alpha$-Curcumene & $\mathrm{C}_{15} \mathrm{H}_{22}$ & [14] \\
\hline 77 & $\beta$-Bisabolene & $\mathrm{C}_{15} \mathrm{H}_{24}$ & [15] \\
\hline 78 & $\beta$-Sesquiphellandrene & $\mathrm{C}_{15} \mathrm{H}_{24}$ & [14] \\
\hline 79 & Nerolidol & $\mathrm{C}_{15} \mathrm{H}_{26} \mathrm{O}$ & [15] \\
\hline 80 & $\beta$-Farnesene & $\mathrm{C}_{15} \mathrm{H}_{24}$ & [15] \\
\hline 81 & $\alpha$-Bergamotene & $\mathrm{C}_{15} \mathrm{H}_{24}$ & [16] \\
\hline 82 & Germacrene D & $\mathrm{C}_{15} \mathrm{H}_{24}$ & [15] \\
\hline 83 & Germacrene B & $\mathrm{C}_{15} \mathrm{H}_{24}$ & [14] \\
\hline 84 & $\alpha$-Bisabolol & $\mathrm{C}_{15} \mathrm{H}_{26} \mathrm{O}$ & {$[14]$} \\
\hline 85 & $\alpha$-Bisabolene & $\mathrm{C}_{15} \mathrm{H}_{24}$ & [14] \\
\hline 86 & $\beta$-Eudesmol & $\mathrm{C}_{15} \mathrm{H}_{26} \mathrm{O}$ & [16] \\
\hline 87 & Cyclosativene & $\mathrm{C}_{15} \mathrm{H}_{24}$ & [14] \\
\hline 88 & $\beta$-Elemol & $\mathrm{C}_{15} \mathrm{H}_{26} \mathrm{O}$ & [14] \\
\hline 89 & $\beta$-Elemene & $\mathrm{C}_{15} \mathrm{H}_{24}$ & [14] \\
\hline 90 & $\delta$-Elemene & $\mathrm{C}_{15} \mathrm{H}_{24}$ & [16] \\
\hline 91 & $\gamma$-Elemene & $\mathrm{C}_{15} \mathrm{H}_{24}$ & [16] \\
\hline 92 & $\gamma$-Eudesmol & $\mathrm{C}_{10} \mathrm{H}_{18} \mathrm{O}$ & [14] \\
\hline 93 & $\alpha$-Eudesmol & $\mathrm{C}_{10} \mathrm{H}_{18} \mathrm{O}$ & [15] \\
\hline 94 & Thujopsene & $\mathrm{C}_{15} \mathrm{H}_{24}$ & [16] \\
\hline
\end{tabular}

researchers found that 6-gingerol $(2 \mathrm{mg} / \mathrm{kg})$ increased cognitive ability and inhibited the over activation of astrocytes. Therefore, the study indicated that 6-gingerol played an effective neuroprotective role, most likely through its antioxidant and anti-inflammatory activities [38].

5.2. Pharmacological Effects on Cardiovascular and Cerebrovascular Systems. Treatment of cardiovascular and cerebrovascular systems is not one of the traditional uses 
TABle 4: Diarylheptanoid compounds isolated from Z. officinale.

\begin{tabular}{|c|c|c|}
\hline No. & Chemical component & Ref. \\
\hline 95 & 5-Hydroxy-1-(3, 4-dihydroxy-5-methoxyphenyl)-7-(4-hydroxy-3-methoxyphenyl)-3-heptanone & [13] \\
\hline 96 & 3-Acetoxy-5-hydroxy-1-(4-hydroxy-3-methoxyphenyl)-7-(3, 4-dihydroxyphenyl) heptanes & [13] \\
\hline 97 & 5-Hydroxy-1, 7-bis (4-hydroxy-3-methoxyphenyl)-3-heptanone & [13] \\
\hline 98 & 5-Hydroxy-1-(4-dihydroxy-3-methoxyphenyl)-7-(3, 4-dihydroxyphenyl)-3-heptanone & [13] \\
\hline 99 & 3, 5-Diacetoxy-7-(4-dihydroxy-3-methoxyphenyl)-1-(3, 4-dihydroxy-5-methoxyphenyl) heptane & [13] \\
\hline 100 & (3S, 5S)-3, 5-Diacetoxy-1, 7-bis(4-hydroxy-3-methoxy-phenyl) heptane & [18] \\
\hline 101 & (3R, 5S)-3-Acetoxy-5-hydroxy-1, 7-bis(4-hydroxy-3-methoxyphenyl) heptane & [18] \\
\hline 102 & (3R, 5S)-3, 5-Dihydroxy-1-(4-hydroxy-3, 5-dimethoxyphenyl)-7-(4-hydroxy-3-methoxyphenyl) heptane & [18] \\
\hline 103 & $(3 \mathrm{R}, 5 \mathrm{~S})-3,5$-Diacetoxy-1,7-bis (4-hydroxy-3-methoxyphenyl) heptane & [18] \\
\hline 104 & (3R, 5S)-3, 5-Diacetoxy-1-(4-hydroxy-3, 5-dimethoxyphenyl)-7-(4-hydroxy-3-methoxy-phenyl) heptane & [18] \\
\hline 105 & (3S, 5S)-3, 5-Dihydroxy-1, 7-bis (4-hydroxy-3-methoxyphenyl) heptane & [18] \\
\hline 106 & (3R,5S)-3,5-Dihydroxy-1,7-bis (4-hydroxy-3-methoxyphenyl) heptane & [18] \\
\hline 107 & (3S, 5S)-3, 5-Dihydroxy-1-(4-hydroxy-3, 5-dimethoxyphenyl)-7-(4-hydroxy-3-methoxyphenyl) heptane & [18] \\
\hline 108 & (5S)-5-Acetoxy-1, 7-bis (4-hydroxy-3-methoxyphenyl)heptan-3-one & [18] \\
\hline 109 & 5-Hydroxy-1-(3, 4-dihydroxy-5-methoxyphenyl)-7-(4-hydroxy-3-methoxyphenyl) heptan-3-one & [18] \\
\hline 110 & 5-Hydroxy-1-(4-hydroxy-3-methoxyphenyl)-7-(3, 4-dihydroxy-5-methoxyphenyl) heptan-3-one & [18] \\
\hline 111 & 5-Hydroxy-1-(4-hydroxy-3-methoxyphenyl)-7-(3, 4-dihydroxyphenyl)heptan-3-one & [18] \\
\hline 112 & 1, 5-Epoxy-3-hydroxy-1-(4-hydroxy-3, 5-dimethoxyphenyl)-7-(4-hydroxy-3-methoxyphenyl) heptane & [18] \\
\hline 113 & 3-Acetoxy-1, 5-epoxy-1-(3, 4-dihydroxy-5-methoxyphenyl)-7-(4-hydroxy-3-methoxy-phenyl) heptane & [18] \\
\hline 114 & 1, 7-Bis (4-hydroxy-3-methoxyphenyl) hept-4-en-3-one & [18] \\
\hline 115 & 1, 5-Epoxy-3S-hydroxy-1-(3, 4-dihydroxy-5-methoxyphenyl)-7-(4-hydroxy-3-methoxyphenyl) heptane & [19] \\
\hline 116 & 1, 5-Epoxy-3R-hydroxy-1-(3, 4-dihydroxy-5-methoxyphenyl)-7-(4-hydroxy-3-methoxyphenyl) heptane & [19] \\
\hline 117 & 3, 5-Diacetyl-1-(3-methoxy-4, 5-dihydroxyphenyl)-7-(4-hydroxy-3-methoxyphenyl) heptane & [19] \\
\hline 118 & 3-Acetoxy-1, 5-epoxy-1-(3, 4-dihydroxy-5-methoxyphenyl)-7-(4-hydroxy-3-methoxyphenyl) heptane & [20] \\
\hline
\end{tabular}
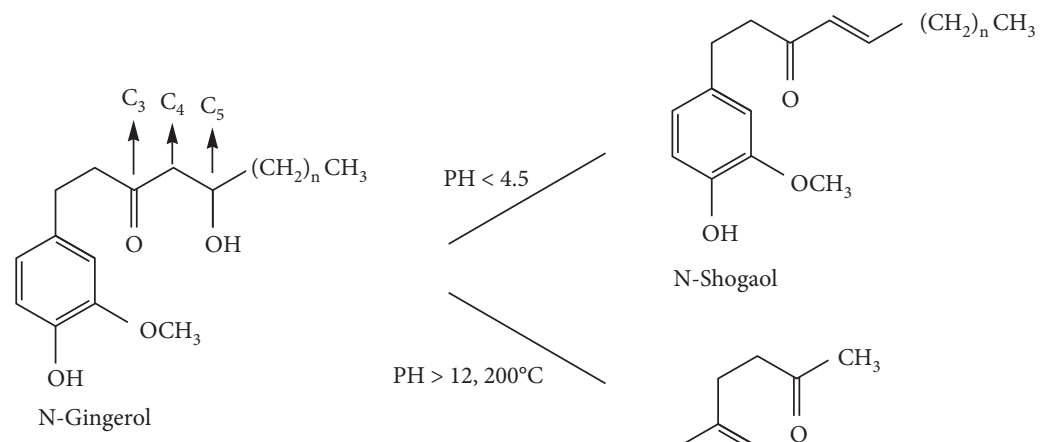

N-Shogaol

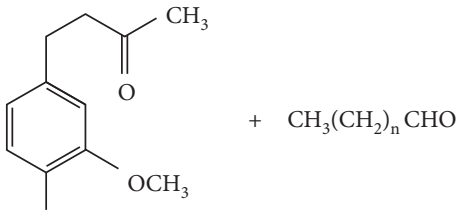

$\mathrm{OH}$

Zingerone

Figure 2: Gingerols and its chemical changes [11].

of ZRR, but they have been identified as potential targets as part of an in-depth study of the pharmacological effects of ZRR. Moreover, with the development of modern separation and purification technology, the compounds in ZRR also have protective effects on the cardiovascular and cerebrovascular systems, such as 6-gingerol. The results showed that its mechanisms are closely related to antioxidant and anti-inflammatory effects.

5.2.1. Cardioprotective Activity. The research conducted by El-Hawwary et al. was aimed at further evaluating the mechanism by which ZRR protected cisplatin-induced myocardial injury. They believed that ZRR protected the cardiotoxicity caused by cisplatin through downregulating the TNF- $\alpha$ and P53 immune expression and reducing the levels of LDH and CK [39].

Further research showed that 6-gingerol reduced the content of serum malondialdehyde and increased superoxide dismutase content. By inhibiting oxidative stress, 6-gingerol alleviated the damage of cardiac myocytes, indicating that 6-gingerol had cardioprotective effect [40]. 6-Gingerol inhibited the apoptosis of AC16 cardiomyocytes induced by ischemia-reperfusion and reduced the level of ROS [41]. The cardioprotective activity of 6-gingerol may be mediated by inhibiting L-type 


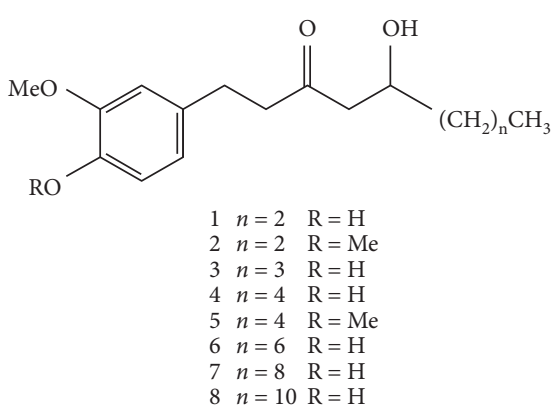

(a)<smiles>[R]c1ccc(CCC(=O)CCCC)cc1OC</smiles>

$$
\begin{array}{lll}
22 & n=6 & \mathrm{R}=\mathrm{H} \\
23 & n=6 & \mathrm{R}=\mathrm{Me} \\
24 & n=8 & \mathrm{R}=\mathrm{H} \\
25 & n=8 & \mathrm{R}=\mathrm{Me} \\
26 & n=10 & \mathrm{R}=\mathrm{H}
\end{array}
$$

(d)<smiles>CCCCCCCC(=O)CC(=O)/C=C/c1ccc(O)c(C(=O)O)c1</smiles>

$$
\begin{array}{ll}
32 & n=4 \\
33 & n=6 \\
34 & n=8 \\
35 & n=10
\end{array}
$$

(g)<smiles>CCCC(CC(=O)CCc1ccc([18O])c(OC)c1)OC(C)=O</smiles>

$$
\begin{array}{rll}
9 & n=2 & \mathrm{R}=\mathrm{H} \\
10 & n=4 & \mathrm{R}=\mathrm{H} \\
11 & n=6 & \mathrm{R}=\mathrm{H} \\
12 & n=8 & \mathrm{R}=\mathrm{H}
\end{array}
$$

(b)<smiles>COc1cc(CCC(C)=O)ccc1O</smiles>

27

(e)<smiles>CCCCC(O)CC(O)CCc1ccc(O)c(OC)c1</smiles>

$$
\begin{array}{ll}
36 & n=2 \\
37 & n=4 \\
38 & n=6 \\
39 & n=8
\end{array}
$$

(h)

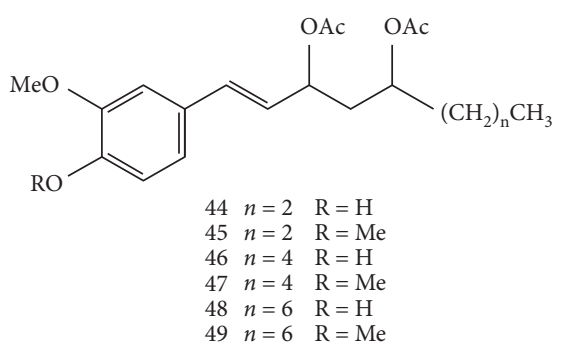<smiles>CCCC=CC(=O)CCc1ccc(OCCC)c(OC)c1</smiles>

(c)<smiles>CCCCCCCCC(=O)CC(=O)CCc1ccc(O)c(OC)c1</smiles>

$$
\begin{array}{ll}
28 & n=4 \\
29 & n=6 \\
30 & n=8 \\
31 & n=10
\end{array}
$$

(f)<smiles>[R6]c1ccc(CCC(O)CC(CCCC)OC(C)=O)cc1OC</smiles>

$$
\begin{array}{lll}
40 & n=2 & \mathrm{R}=\mathrm{H} \\
41 & n=2 & \mathrm{R}=\mathrm{Me} \\
42 & n=4 & \mathrm{R}=\mathrm{H} \\
43 & n=4 & \mathrm{R}=\mathrm{Me}
\end{array}
$$

(i)

(j)

FIgURE 3: The structures of gingerols from $Z$. officinale.

$\mathrm{Ca}^{2+}$ in rat cardiomyocytes and reducing extracellular $\mathrm{Ca}^{2+}$ influx to reduce intracellular $\mathrm{Ca}^{2+}[42]$.

Investigation of the recovery of regional myocardial function in thoracotomy before a 30-minute left anterior descending coronary artery ligation, given pretreatment with 6-gingerol at a dose of $6 \mathrm{mg} / \mathrm{kg}$ (intravenous injection), 6-gingerol pretreatment decreased the level of markers of myocardial injury, which demonstrated that 6-gingerol protected myocardium to a certain extent [43].

When Ren et al. studied the protective mechanism of 6gingerol on cardiomyocytes $\mathrm{H} 9 \mathrm{c} 2$ cells, they found that 6gingerol had cytoprotective effect on hypoxia-induced injury in a concentration-dependent manner at a lower concentration $(5-50 \mu \mathrm{m})$. Their experimental results showed that 6- gingerol inhibited the expression of BNIP3 and activated the $\mathrm{PI} 3 \mathrm{~K} / \mathrm{AKT} / \mathrm{mTOR}$ signaling pathway, which resulted in the inhibition of apoptosis and autophagy [44]. Whether there are other mechanisms of cardioprotective activity of ZRR requires further study.

5.2.2. Cerebral Ischaemia Protection. Studies have found that ZRR has protective effects against focal cerebral ischemia-reperfusion (FCI-R) injury. For example, total phenol of ZRR was reported to improve the neurological symptoms of MCAO rats, prolonged the passive conditioned reflex latency and reduced the number of errors, and significantly reduced the area of cerebral infarction. Brain 


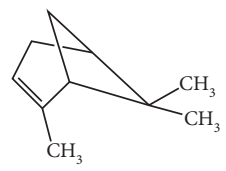

50

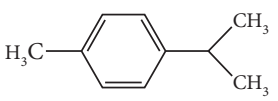

55<smiles>C=C1CCC2CC1C2(C)C</smiles>

60

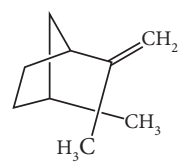

51<smiles>CC1=CC=C(C(C)C)CC1</smiles>

56<smiles>CC1=CCC(C(C)C)C=C1</smiles>

61<smiles>C=CC(=C)CCC=C(C)C</smiles>

52<smiles>CC(C)=CCCC(C)=CCO</smiles>

57

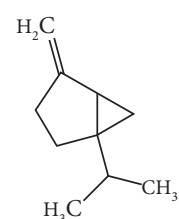<smiles>C=C1C=CC(C(C)C)CC1</smiles><smiles>CC1=CCC(C(C)(C)O)CC1</smiles><smiles>CC(C)=CCCC(C)CC=O</smiles>

63

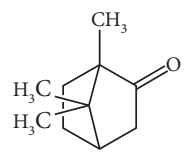

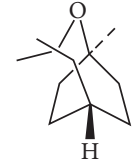

54

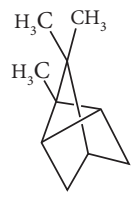<smiles>CC1=CCC(=C(C)C)CC1</smiles>

64

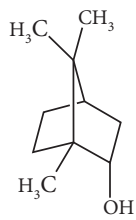<smiles>CC(C)=CCCC(C)CCO</smiles>

70<smiles>CC(C)=CCC/C(C)=C/C=O</smiles><smiles>CC(C)=CCCC(C)=CC(=O)O</smiles>

72<smiles>CC(=O)OCCC(C)CCC=C(C)C</smiles><smiles>CC(=O)OC/C=C(\C)CCC=C(C)C</smiles>

(a)

FIgURE 4: Continued. 
<smiles>CC(C)=CCCC(C)C1C=CC(C)=CC1</smiles>

75<smiles>C=CC(=C)CCC=C(C)CCC=C(C)C</smiles>

80<smiles>CC(C)=CCC[C@](C)(O)[C@H]1CC=C(C)CC1</smiles>
84<smiles>C=C[C@]1(C)CC[C@@H](C(C)(C)O)C[C@H]1C(=C)C</smiles>

88<smiles>CC1=C2CC(C(C)(C)O)CCC2(C)CCC1</smiles>

92<smiles>CC(C)=CCCC(C)c1ccccc1</smiles>

76<smiles>C=C(CCC=C(C)C)C1CC=C(C)CC1</smiles>

77<smiles>CC(C)=CCCC(C)c1ccc(C)cc1</smiles><smiles>C=CC(C)(O)CCC=C(C)CCC=C(C)C</smiles>

79<smiles>CC(C)=CCCC(C)=CCCC(C)C</smiles>

83

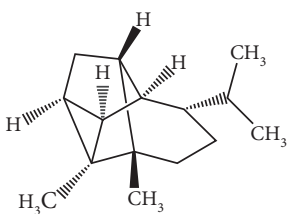

87<smiles>C=C1CCC[C@]2(C)CC[C@@H](C(C)(C)O)C[C@@H]12</smiles>

86<smiles>CC(C)=CCC=C(C)C1CC=C(Cl)CC1</smiles>

85<smiles>C=CC1(C)CCC(C(C)C)=CC1C(=C)C</smiles>

90<smiles>C=CC1(C)CCC(=C(C)C)CC1C(=C)C</smiles>

91

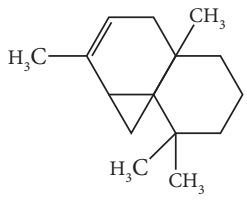

94

(b)

Figure 4: The structures of essential oils from Z. officinale: (a) monoterpenoids; (b) sesquiterpenoids.

histopathological examination revealed that the number of bleeding cases and neutrophils in the treated group were significantly lower than untreated group [45]. Jia et al. showed that the protective effect of ZAE on FCI-R injury could be attributed to apoptosis signal relative protein. ZAE significantly reduced caspase- 3 gene expression, decreased Bax and Bcl-2 positive cells, and increased the ratio of Bcl-2/Bax [46].

In addition to FCI-R injury, ZRR also had a protective effect on cerebral ischemia-reperfusion (CI-R) injury. The experimental results of Wang et al. confirmed the protective effect of ZAE $(100 \mathrm{mg} / \mathrm{kg})$ on CI-R injury, which seems to be associated with inflammatory factors and adhesion factors, involving changes in the in vivo activity of TNF- $\alpha$, ICAM-1, and P-selectin [47]. ZAE $(0.2 \mathrm{~g} / \mathrm{kg})$ significantly mitigated brain tissue edema in rats, prolonged thrombin time, and reduced plasma fibrinogen content. It suggested that protective effect of ZAE is partially related to its anticoagulant effect [48]. 
<smiles>[R3]c1cc(CCC(O)CC(=O)CCc2cc([R])c([R])c(O)c2)ccc1O</smiles>

$\begin{array}{lll}\mathrm{R}_{1} & \mathrm{R}_{2} & \mathrm{R}_{3}\end{array}$ $95 \mathrm{CH}_{3} \mathrm{O} \quad \mathrm{OH} \quad \mathrm{CH}_{3} \mathrm{O}$

$96 \quad \mathrm{OH} \quad \mathrm{CH}_{3} \mathrm{O} \quad \mathrm{OH}$<smiles>COc1cc(C(CC(O)CC(C)=O)c2ccc(O)c(OC)c2)ccc1O</smiles>

98<smiles>[R6]c1ccc(CCC([R6])CC([R9])CCc2cc([R9])c([R6])c(OC)c2)cc1O</smiles>

$\begin{array}{lllll}\mathrm{R}_{1} & \mathrm{R}_{2} & \mathrm{R}_{3} & \mathrm{R}_{4} & \mathrm{C}-3\end{array}$

$\begin{array}{llllll}100 & \text { Ac } & \text { Ac } & \text { H } & \text { H } & \text { S }\end{array}$

$\begin{array}{llllll}101 & \mathrm{Ac} & \mathrm{H} & \mathrm{H} & \mathrm{H} & \mathrm{R}\end{array}$

$102 \mathrm{H} \quad \mathrm{H} \quad \mathrm{H}^{\circ \mathrm{OCH}_{3}} \mathrm{R}$

103 Ac Ac $\mathrm{H} \quad \mathrm{H} \quad \mathrm{R}$

$104 \mathrm{Ac} \quad \mathrm{Ac} \quad \mathrm{H} \quad \mathrm{OCH}_{3} \quad \mathrm{R}$

$\begin{array}{lllllll}105 & \mathrm{H} & \mathrm{H} & \mathrm{H} & \mathrm{H} & \mathrm{R}\end{array}$

$\begin{array}{lllllll}106 & \mathrm{H} & \mathrm{H} & \mathrm{H} & \mathrm{H} & \mathrm{R}\end{array}$

$\begin{array}{lllllllll}107 & \mathrm{H} & \mathrm{H} & \mathrm{H} & \mathrm{OCH}_{3} & \mathrm{~S}\end{array}$<smiles>COc1cc(CCCOC2CC(O)CC(c3cc(C)c(O)c(OC)c3)C2)ccc1O</smiles>

112<smiles>[R]c1cc(CCC(O)CC([R3])CCc2ccc(O)c(OC)c2)cc([R])c1O</smiles>

$97 \mathrm{R}_{1}=\mathrm{H}, \mathrm{R}_{2}=\mathrm{OH}, \mathrm{R}_{3}=\mathrm{CH}_{3} \mathrm{O}_{2}$<smiles>COc1cc(CCC(CC(CCc2ccc(O)c(OC)c2O)OC(C)=O)OC(C)=O)cc(O)c1O</smiles>

99<smiles>[R6]c1cc(CCC(CC(=O)CCc2cc([R6])c(O)c([R6])c2)C(=O)OCc2ccccc2)cc([2H])c1[O-]</smiles>

$\begin{array}{lllllll}\mathrm{R}_{1} & \mathrm{R}_{2} & \mathrm{R}_{3} & \mathrm{R}_{4} & \mathrm{R}_{5} & \mathrm{R}_{6} & \mathrm{R}_{7}\end{array}$ $\begin{array}{cccccccc}108 & \mathrm{CH}_{3} & \mathrm{CH}_{3} & \mathrm{H} & \mathrm{H} & \mathrm{H} & \mathrm{H} & \mathrm{Ac} \\ 109 & \mathrm{H} & \mathrm{CH}_{3} & \mathrm{H} & \mathrm{H} & \mathrm{OCH}_{3} & \mathrm{H} & \mathrm{H}\end{array}$ $\begin{array}{llllllllll}110 & \mathrm{CH}_{3} & \mathrm{H} & \mathrm{H} & \mathrm{H} & \mathrm{H} & \mathrm{OCH}_{3} & \mathrm{Ac}\end{array}$ $\begin{array}{lllllllll}111 & \mathrm{CH}_{3} & \mathrm{H} & \mathrm{H} & \mathrm{H} & \mathrm{H} & \mathrm{H} & \mathrm{H}\end{array}$<smiles>COc1cc(CCCOC(CC2CC2C(=O)O)c2cc(O)c(O)c(CO)c2)ccc1O</smiles>

113<smiles>COc1cc(CC/C=C/C(=O)CCc2ccc(O)c(CO)c2)ccc1O</smiles>

114

Figure 5: The structures of diarylheptanoids from Z. officinale. 
TABLE 5: Pharmacological action of ZE or certain ingredients.

\begin{tabular}{|c|c|c|c|c|c|}
\hline $\begin{array}{l}\text { Pharmacological } \\
\text { activity }\end{array}$ & Assay & Subject & Treatment & Effect & Ref. \\
\hline Treatment of $\mathrm{AD}$ & $\begin{array}{c}\text { The Morris water maze } \\
\text { test }\end{array}$ & $\begin{array}{c}A \beta_{25-35} \text { and } \\
\text { aluminum chloride } \\
\text { induced memory } \\
\text { impairment rat } \\
\text { model }\end{array}$ & $\mathrm{ZAE}(1,2$, and $4 \mathrm{~g} / \mathrm{kg})$ & $\begin{array}{c}\text { The latency of water maze } \\
\text { test shortened. }\end{array}$ & {$[25]$} \\
\hline $\begin{array}{l}\text { Improving } \\
\text { cognition }\end{array}$ & $\begin{array}{l}\text { Novel object } \\
\text { recognition test }\end{array}$ & $\begin{array}{l}\text { A mice model of } \\
\text { scopolamine- } \\
\text { induced memory } \\
\text { deficits }\end{array}$ & ZSE $(5,25$, and $125 \mathrm{mg} / \mathrm{kg})$ & $\begin{array}{l}\text { ZE induced the upregulation } \\
\text { of NGF and increased the } \\
\text { levels of ERK and CREB in } \\
\text { the hippocampus. }\end{array}$ & {$[26]$} \\
\hline $\begin{array}{l}\text { Cardioprotective } \\
\text { activity }\end{array}$ & $\begin{array}{l}\text { Cardioprotective } \\
\text { activity evaluation test }\end{array}$ & $\begin{array}{l}\text { Rat ventricular } \\
\text { myocytes }\end{array}$ & $\begin{array}{l}\text { 6-Gingerol }(3,10,30,100, \text { and } \\
300 \mu \mathrm{mol} / \mathrm{L})\end{array}$ & $\begin{array}{l}\text { 6-Gingerol reduced the L- } \\
\text { type } \mathrm{Ca}^{2+} \text { in a concentration- } \\
\text { dependent manner with a } \\
\text { half-maximal inhibitory } \\
\text { concentration }\left(\mathrm{IC}_{50}\right) \text { of } \\
31.25 \mu \mathrm{mol} / \mathrm{L} \text { in } \\
\text { cardiomyocytes. }\end{array}$ & {$[27]$} \\
\hline $\begin{array}{l}\text { Cerebral ischaemia } \\
\text { protection }\end{array}$ & $\begin{array}{l}\text { Cerebral ischemia- } \\
\text { reperfusion assay }\end{array}$ & Rats & ZAE $(200,100$, and $50 \mathrm{mg} / \mathrm{kg})$ & $\begin{array}{l}\text { ZAE decreased the contents } \\
\text { of } \mathrm{Glu}, \mathrm{Ca}^{2+} \text { and water in } \\
\text { brain tissue and increased } \\
\text { the activities of SOD. }\end{array}$ & {$[28]$} \\
\hline Antiemetic effect & Intestinal tension test & $\begin{array}{l}\text { Isolated guinea pig } \\
\text { ileum }\end{array}$ & $\begin{array}{c}\text { 6-Gingerol and 6-shogaol } \\
\left(3 \times 10^{-5}, 1 \times 10^{-5}, 3 \times 10^{-6} \mathrm{~mol} / \mathrm{L}\right)\end{array}$ & $\begin{array}{l}\text { They dose-dependently } \\
\text { inhibited the increase of } \\
\text { contraction of the guinea pig } \\
\text { ileum stimulated by 5-HT } \\
\text { and 2-me-5-HT, reduced the } \\
\text { mean tension. }\end{array}$ & {$[29]$} \\
\hline $\begin{array}{l}\text { Antimicrobial } \\
\text { effect }\end{array}$ & $\begin{array}{l}\text { The KB disc diffusion } \\
\text { method }\end{array}$ & $\begin{array}{c}\text { Aspergillus flavus } \\
\text { and Aspergillus } \\
\text { niger }\end{array}$ & ZEE & $\begin{array}{c}\text { The antibacterial diameter of } \\
\text { ZEE to Aspergillus niger was } \\
21.80 \mathrm{~mm} \text { and to Aspergillus } \\
\text { flavus was } 16.83 \mathrm{~mm} \text {. }\end{array}$ & {$[30]$} \\
\hline Antitumour effect & $\begin{array}{l}\text { MTT cell proliferation } \\
\text { assay; comet assay } \\
\text { (single-cell gel } \\
\text { electrophoresis) }\end{array}$ & $\begin{array}{c}\text { Human colon } \\
\text { cancer cell line, } \\
\text { HCT116 }\end{array}$ & $\begin{array}{l}\text { Zingerone }(\mathrm{ZO})(2.5,5 \text {, and } \\
\qquad 10 \mu \mathrm{M})\end{array}$ & $\begin{array}{l}\text { ZO drastically reduced cell } \\
\text { proliferation and increased } \\
\text { the percentage of DNA } \\
\text { damage in HCT116 cells. }\end{array}$ & {$[31]$} \\
\hline $\begin{array}{l}\text { Anti- } \\
\text { inflammatory } \\
\text { effect }\end{array}$ & $\begin{array}{l}\text { In vivo anti- } \\
\text { inflammatory effect test }\end{array}$ & $\begin{array}{l}\text { 5-week-old female } \\
\text { mice }\end{array}$ & ZEE $(100,300$, and $500 \mathrm{mg} / \mathrm{kg})$ & $\begin{array}{l}\text { Body weight, colon length, } \\
\text { and DAI values of mice } \\
\text { improved. }\end{array}$ & {$[32]$} \\
\hline $\begin{array}{l}\text { Anti-oxidative } \\
\text { effect }\end{array}$ & $\begin{array}{l}\text { DPPH and ferric } \\
\text { reducing antioxidant } \\
\text { properties (FRAP) } \\
\text { assay }\end{array}$ & Albino rats & ZME $(2.5-100 \mu \mathrm{g} / \mathrm{mL})$ & $\begin{array}{l}\text { The extract promoted an } \\
\text { inhibition of free radicals } \\
\text { with } \text { IC }_{50} \text { values of } \\
47.05 \pm 2.03 \mu \mathrm{g} / \mathrm{mL} \text { and } \\
89.15 \pm 0.29 \mu \mathrm{g} / \mathrm{mL} \text { in DPPH } \\
\text { and FRAP assay. }\end{array}$ & {$[33]$} \\
\hline
\end{tabular}

In the treatment of CI-R, polysaccharide of ZRR enhanced the SOD activity of brain tissue to inhibit the production of free radicals, promote lipid peroxidation, and reduce the production of MDA. In addition, polysaccharide of ZRR has been shown to reduce the content of $\mathrm{NO}$ in brain tissue [49].

5.3. Antiemetic Effect. ZRR is a traditional Chinese medicine (TCM) widely used to relieve vomiting in Asian countries. Many experts and scholars have studied the antiemetic effect of ZRR. The antiemetic effect of ZRR may be related to the 5$\mathrm{HT}_{3}$ receptor, $\mathrm{M}_{3}$ cholinocepter in gastrointestinal tract [50], and $\mathrm{NK}_{1}$ receptor [51]. For example, Walstab et al. found that ZSE inhibited $\mathrm{Ca}^{2+}$ influx of $5-\mathrm{HT}_{3}$ receptor, and noncompetitively inhibited the activation of human $5-\mathrm{HT}_{3}$ receptor [52]. Some scholars have studied the effects of ZAE $(600,300$, and $150 \mathrm{mg} / \mathrm{kg})$ on vomiting in rats and found that ZAE had antiemetic effect, and its mechanism may be related to substance P, 5-HT, and the vomiting center in the central nervous system, which provided a basis for investigations into the use of the ZRR as a drug [53].

Some scholars have also studied the pharmacological effects of different processed products of ZRR on vomiting. Ginger juice $(4 \mathrm{~g} / \mathrm{kg}, 2 \mathrm{~g} / \mathrm{kg})$ and ginger decoction $(10 \mathrm{~g} / \mathrm{kg}$, $5 \mathrm{~g} / \mathrm{kg}$ ) could reduce the amount of kaolin intake, reflecting ginger had antiemetic effect. The ginger juice low-dose group $(2 \mathrm{~g} / \mathrm{kg})$ had the best antiemetic effect, and its antiemetic substance may be 6-gingerol [54]. In an experiment comparing the effect of dried ginger juice (DGJ), fresh ginger 
juice (FGJ), and fresh ginger boiled juice (FGBJ) in relieving the emetic effects, the study found that the above ginger juice groups reduced the intake of kaolin compared with the cisplatin group, indicating that all kinds of ginger juice could be effective prevent vomiting [10].

In addition to the above mentioned pharmacological effects, research has been conducted into the medicinal effects of ingredients isolated from ZRR, such as 6-gingerol and 6-shogaol. For example, in a study about the effects of 6gingerol and 6-shogaol on isolated guinea pig ileum contraction, the study found that the mechanism of antiemetic may be noncompetitive blocking of the $5-\mathrm{HT}_{3}$ receptor by 6 gingerol and 6-shogaol [29]. 6-Gingerol and 6-shogaol could also noncompetitively block the $\mathrm{NK}_{1}$ receptor, which suggested that 6-gingerol and 6-shogaol may be the main active ingredients of ZRR for alleviating vomiting [51].

ZRR has been widely used for nausea and vomiting since ancient times, and modern research has also confirmed the antiemetic effect of ZRR during pregnancy, chemotherapy, and surgery. For pregnant women with mild to moderate nausea and vomiting in the early stage of pregnancy, ZRR $(500 \mathrm{mg})$ and vitamin B6 (40 mg) were administered twice a day, and the effect is equivalent. However, ZRR is more effective for severity of nausea and amount of vomiting [55].

In an experiment, forty-seven patients with gynecological malignancies were enrolled for chemotherapy with carboplatin and paclitaxel. The results showed that ZRR significantly reduced the nausea and vomiting of the patients. However, the results of delayed and acute phase were still unclear [56].

Chang et. concluded oral administration of $0.5-2.0 \mathrm{~g} / \mathrm{d}$ ZE mitigated acute CINV in patients, especially acute vomiting [57]. Konmun et al. found that a major bioactive constituent of ZRR, 6-gingerol, exerted antiemetic effects in patients who received moderately to highly emetogenic adjuvant chemotherapy [58]. In one experiment, 100 patients with cholelithiasis who were candidated for laparoscopic cholecystectomy were treated using TCM Zingiberis Rhizoma Recens (500 mg) as the treated group, using the Western medicine ondansetron $(4 \mathrm{mg})$ as a control. The results showed that the incidence of postoperative nausea in the treated group was significantly lower than the ondansetron group, indicating that the treatment of PONV with ZRR improved symptom to a certain extent [59].

5.4. Antimicrobial Effect. Many in vitro antibacterial studies have shown that ZRR had broad-spectrum antibacterial effects. The volatile oil from ZSE had an antibacterial effect on many kinds of bacteria, not only on the Gram-positive bacteria such as Staphylococcus aureus and Bacillus subtilis, but also on the Gram-negative bacteria such as Escherichia coli and Salmonella. In addition, volatile oil from ZRR had a stronger inhibiting ability to fungus compared with bacteria [60].

ZEE has been reported to have antimicrobial effects against Pseudomonas aeruginosa, Escherichia coli, Staphylococcus aureus, Klebsiella pneumoniae, Bacillus cereus,
Acinetobacter baumannii, Candida albicans, and Candida krusei. The MICs of ZRR against the above bacteria are 40, $40,20,20,20,20$, and $10,5 \mathrm{mg} / \mathrm{mL}$, respectively [61]. In broth microdilution assays, ZME exhibited antibacterial activities against Klebsiella pneumoniae ( $\mathrm{MIC}=12.5 \mu \mathrm{g} / \mathrm{mL}$ ). With the increase of extract concentration, the inhibition zone increased. When the concentration of the extract was $100 \mu \mathrm{g} / \mathrm{mL}$, Klebsiella pneumonia showed the highest susceptibility $(29.04 \pm 0.35 \mathrm{~mm})$, followed by Pseudomonas aeruginosa, Escherichia coli, and Salmonella typhi, while Staphylococcus aureus had the least inhibition zone [33]. Different concentration of ZEE had different degrees of antibacterial effect, and the most antibacterial effect was at $75 \%$ ethanol solution. And the effect was as follows: Bacillus subtilis $>$ Yeast $>$ Staphylococcus albus $>$ Escherichia coli $>$ mixed microbes from egg surface [62].

Furthermore, essential oils, polyphenols, flavonoids, and gingerols in ZRR also had antimicrobial effects. The resazurin microtiter assay plate and broth microdilution method were used to investigate the antibacterial effect of ZRR in vitro. It was found that volatile oil from ZRR inhibited Mycobacterium tuberculosis and Nontuberculous mycobacteria [14]. The inhibition rate of polyphenol from ZRR to Escherichia coli was 75\% [63]. Flavonoids from ZRR had different degrees of inhibitory effect on the five kinds of bacteria, and the order of the inhibitory effect was Bacillus subtilis $>$ Aspergillus niger $>$ Staphylococcus aureus $>$ Penicillium $>$ Escherichia coli [64]. In the range of test concentration, gingerols had antibacterial activity against Gram-negative and Gram-positive bacteria, but the antibacterial effect is moderately sensitive $(10-15 \mathrm{~mm})$, and the antibacterial activity decreased with the decrease of gingerol concentration [65].

5.5. Antitumour Effect. ZAE $(100 \mathrm{mg} / \mathrm{kg})$ significantly inhibited gastric cancer in $\mathrm{N}$-nitroso $\mathrm{N}$-methyl urea (MNU)induced albino Wistar rats. The inhibitory effect was associated with combating pathological changes such as inflammation and oxidative stress [66]. ZEE inhibited the growth and proliferation of $\mathrm{TM}_{4}$ cell (Sertoli cells of normal mouse testis). As the treatment concentration increased, this inhibitory effect became stronger and stronger in a dosedependent manner. When the treatment concentration reached $100 \mu \mathrm{g} / \mathrm{mL}$, the growth and proliferation of $\mathrm{TM}_{4}$ cells had been basically inhibited [67]. The crude flavonoid of ZE inhibited the proliferation of the hepatocellular carcinoma cell line HepG2 in a time and dose-dependent manner and induced HepG2 cell apoptosis through a mitochondriamediated apoptosis pathway [68].

As chemical extraction and separation technology progresses, research on the anticancer effects of ZRR has been concentrated in several major components of ZRR, such as 6-gingerol, 6-shogaol, and other ingredients.

According to reports in the past years, 6-gingerol has been proven to have potential anticancer activities against renal-cell carcinoma [69], human oral (SCC4, KB) and cervical cancer (HeLa) cell lines [70], human colon cancer 
cell LoVo [71], human breast cancer MCF-7 and MDA-MB231 [72], human umbilical vein endothelial cells [73], osteosarcoma cells [74], and human gastric adenocarcinoma cell line [75]. Its mechanisms included inhibiting the growth of tumour cells, inducing cell cycle arrest and inducing apoptosis. The antitumor effect of 6-gingerol on renal cancer cells in vivo and in vitro showed that 6 -gingerol inhibited the growth of ACHN, 786-O, and 769-P cells in a time- and dose-dependent manner mainly through the AKT-GSK $3 \beta$ cyclin D1 signaling pathway to induce G1 cell cycle arrest and decrease Ki-67 expression in the nucleus [69]. Additionally, 6-gingerol exerted an antitumor effect by inducing cell apoptosis and inducing cell cycle arrest, including G2 phase arrest of $\mathrm{KB}$ and HeLa cells, and $\mathrm{S}$ phase arrest of SCC4 cells [70].

6-Shogaol has been demonstrated to have potential anticancer activities towards human renal-cell carcinoma 786-O [76] and ovarian cancer cell lines A2780 [77], and head and neck squamous cell carcinoma cell lines [78]. Studies showed that 6-shogaol had a dose-dependent antiproliferative effect and induced the apoptosis of head and neck squamous cell carcinoma cell lines by downregulating survivin [78]. 6-Gingerol inhibited STAT-3 translocation of ovarian cancer cells to inhibit cell proliferation and induce apoptosis [77].

Additionally, zingerone, one of the major active phenolic agents of ZRR, significantly reduced cell viability, improved the production of reactive oxygen species, and induced apoptosis of breast cancer cell line MCF-7 [79]. Zingerone reduced the expression of cyclin D1, induced the cleavage of caspase-3 and PARP-1, which arrested mitosis, thereby inhibiting the growth of human neuroblastoma cells [80]. Yet, the anticancer effects of ZRR have been studied insufficiently, and more pharmacological and mechanistic research is required.

5.6. Anti-Inflammatory Effect. ZME showed potential antiarthritis effects in membrane stabilization assay, proteinase inhibitory assay, and protein denaturation inhibition assays. The inhibition rates in the above three experiments were $84.72 \pm 1.38 \%, 82.72 \pm 1.48 \%$, and $81.68 \pm 1.66 \%$, respectively [81]. When Ezzat et al. investigated the anti-inflammatory effect of ZAE on carrageenan-induced rat paw oedema in rats, they found that ZAE exerted an anti-inflammatory property by reducing PGE2 level and improving inflammatory markers [82].

Several constituents isolated from ZRR have been demonstrated to ameliorate a number of inflammatory responses. 6-Shogaol appeared to alleviate the lung inflammation of mice induced by house dust mite antigen in vivo [83]. 6-Gingerol prevented chronic ulcerative colitis through downregulating NF-kB (p65) and suppressing proinflammatory cytokines [84]. In addition to chronic colitis, 6-gingerol ameliorated acute colitis by activating adenosine adenosine monophosphate-activated protein kinase [85].

When exploring the anti-inflammatory mechanism of ZRR and its active ingredients, it was found that 6-shogaol
$(20 \mu \mathrm{g})$ inhibited ultraviolet radiation B-induced inflammatory response, including reducing the expression of COX-2 and iNOS and reducing the expression of IL-6, IL-10, and TNF- $\alpha$ in human epidermal keratinocytes [86]. A study of 6-shogaol showed that it inhibited the inflammatory response of the ligated periodontitis model in mice, and 6shogaol attenuated the inflammatory response by reducing the number of macrophages and neutrophils in periodontal tissues and reducing the expression of IL- $1 \beta$ and TNF- $\alpha$ and, therefore, may be a therapeutic agent against periodontitis [87]. Given that 6-gingerol reduced PGE2 levels and inhibited inflammation-related osteoclast differentiation, 6gingerol could be used as a potential treatment for inflammatory bone loss [88].

In summary, the extracts and isolates of ZRR have antiinflammatory effects. Furthermore, on the basis of defined mechanisms and safety profiles, ZRR has the potential to be developed as new anti-inflammatory drugs.

5.7. Antioxidative Effect. In DPPH free radical scavenging activity, $\beta$-carotene bleaching test, ferric reducing power determination assay, and ZME showed stronger antioxidant activity than ZAE. In addition, the antioxidant activity of ZE was evaluated by the determination of thiobarbituric acid reactive substances and malondialdehyde dosage [89].

Some pharmacological effects of ZRR are achieved through antioxidant action. For example, Mohammed conducted experiments to evaluate whether ZEE effectively protect the thyroid oxidative damage induced by bisphenol $\mathrm{A}$ in male rats. The authors proposed that ZEE protected the thyroid oxidative damage and thyroid hormone disorder by activating $\mathrm{Nrf} 2 / \mathrm{HO}-1$ gene expression and promoting the synthesis of thyroxine [90]. In a model of chronic ulcerative colitis in mice induced by dextran sodium sulfate, 6-gingerol effectively protected the colon from oxidative damage by enhancing the antioxidant capacity of cells and inhibiting lipid peroxidation [84]. The protective effect of $\mathrm{ZE}$ on human chondrocytes induced by IL- $1 \beta$ could be due to its antioxidant properties to lead to a reduction in oxidative stress [91]. Ginger oleoresin $\left(10^{-4} \mathrm{~g} / \mathrm{mL}\right)$ reduced ionizing radiation-induced damage to human mesenchymal stem cells by reducing ROS production and altering nuclear translocation of Nrf2 [92]. Therefore, the antioxidant effect of Zingiberis Rhizoma Recens is not a single pharmacological effect but is closely related to other effects and is one of the possible mechanisms for the realisation of other pharmacological effects.

\section{Toxicology}

Modern toxicology studies have shown that ZRR has no oral toxicity and no genotoxicity. Part of the experimental results and data of the ZRR are listed in Table 6.

For ZRR, the toxicity of various extracts was evaluated. The acute toxicity test showed that the methanol extract and the aqueous extract ( 2 times/day) were fed for 7 days, and no abnormal changes were observed, and all the mice survived. Compared with male mice, the recovery of female mice was slower [93]. The oral toxicity evaluation showed that after 
TABle 6: Toxicological studies of ZRR.

\begin{tabular}{|c|c|c|c|c|}
\hline Assay & Subject & Treatment & Effect & Ref. \\
\hline Oral toxicity test & Mice & $\begin{array}{l}\mathrm{ZAE} \text { and } \mathrm{ZME}(2,4,6,8 \text {, and } \\
10 \mathrm{~g} / \mathrm{kg})\end{array}$ & $\begin{array}{l}\text { No toxic effects were observed at a higher } \\
\text { dose of } 10 \mathrm{~g} / \mathrm{kg} \text { body weight. }\end{array}$ & [89] \\
\hline $\begin{array}{l}\text { Maximum tolerance } \\
\text { test }\end{array}$ & Male and female mice & ZAE and ZEE & $\begin{array}{l}\text { The maximum tolerance of } \mathrm{ZE} \text { on male } \\
\text { and female mice was greater than } \\
66.67 \mathrm{~g} / \mathrm{kg} \text {. }\end{array}$ & {$[93]$} \\
\hline $\begin{array}{l}\text { Acute toxicity test (a } \\
14 \text {-day study) }\end{array}$ & Healthy Sprague-Dawley rats & ZEE $(5000 \mathrm{mg} / \mathrm{kg})$ & $\begin{array}{c}\text { No evidence of toxicity and death was } \\
\text { observed in all rats. }\end{array}$ & \\
\hline $\begin{array}{l}\text { Subacute toxicity test } \\
\text { (a 28-day study) }\end{array}$ & & $\begin{array}{l}\text { ZEE }(500,1000, \text { and } \\
2000 \mathrm{mg} / \mathrm{kg})\end{array}$ & At different doses, only significant & {$[94]$} \\
\hline $\begin{array}{l}\text { Chronic toxicity test (a } \\
12 \text {-month study) }\end{array}$ & & $\begin{array}{l}\mathrm{ZEE}(250,500, \text { and } \\
1000 \mathrm{mg} / \mathrm{kg})\end{array}$ & $\begin{array}{l}\text { differences in hematological and } \\
\text { biochemistry parameters were observed. }\end{array}$ & \\
\hline Cell viability assay & $\begin{array}{l}\text { All cell lines of hepatoma } \\
\text { (HepG2), breast carcinoma } \\
\text { (MDA-MB-231), and lung } \\
\text { adenocarcinoma (A549) }\end{array}$ & ZSE $(20-100 \mu \mathrm{m})$ & $\begin{array}{l}\text { Compared with the control cells, the cell } \\
\text { viability of all cancer cells was } \\
\text { significantly lost. }\end{array}$ & {$[16]$} \\
\hline $\begin{array}{l}\text { Bone marrow } \\
\text { chromosomal } \\
\text { aberration test (a 7-day } \\
\text { study) }\end{array}$ & Male Wistar rats & ZAE (100 mg/kg, 200 mg/kg) & $\begin{array}{l}\text { The chromosome structure of bone } \\
\text { marrow cells was not degraded. }\end{array}$ & {$[95]$} \\
\hline Micronuclei tests & & & $\begin{array}{l}\text { ZAE showed a more structured layout } \\
\text { and healthy polychromatic erythrocyte. }\end{array}$ & \\
\hline $\begin{array}{l}\text { Reproductive toxicity } \\
\text { test (a 28-day study) }\end{array}$ & Adult male Sprague-Dawley rats & ZEE $(1 \mathrm{~g} / \mathrm{kg})$ & $\begin{array}{l}\text { Hormone levels, trace elements, and } \\
\text { antioxidant enzyme activity increased, } \\
\text { while the contents of total homocysteine } \\
\text { and malondialdehyde decreased. }\end{array}$ & {$[96]$} \\
\hline
\end{tabular}

TABLe 7: The quality control of $Z$. officinale in Pharmacopoeia of different countries.

\begin{tabular}{|c|c|c|c|c|c|c|c|c|c|}
\hline \multirow[b]{2}{*}{ Name } & \multirow[b]{2}{*}{$\begin{array}{c}\text { Medicinal } \\
\text { part }\end{array}$} & \multirow[b]{2}{*}{ Pharmacopeia } & \multirow{2}{*}{$\begin{array}{l}\text { Water } \\
\text { detection } \\
\text { or loss on } \\
\text { drying }\end{array}$} & \multicolumn{2}{|c|}{ Ash detection } & \multirow[b]{2}{*}{$\begin{array}{c}\text { Volatile oil } \\
\text { content }\end{array}$} & \multirow[b]{2}{*}{$\begin{array}{l}\text { Extract } \\
\text { content }\end{array}$} & \multicolumn{2}{|c|}{ Determination of content } \\
\hline & & & & $\begin{array}{l}\text { Total } \\
\text { ash }\end{array}$ & $\begin{array}{c}\text { Acid } \\
\text { insoluble } \\
\text { ash }\end{array}$ & & & $\begin{array}{c}\text { Marker } \\
\text { components }\end{array}$ & $\begin{array}{c}\text { Content } \\
\text { requirements }\end{array}$ \\
\hline $\begin{array}{l}\text { Zingiberis } \\
\text { Rhizoma } \\
\text { Recens }\end{array}$ & $\begin{array}{l}\text { Fresh } \\
\text { rhizome }\end{array}$ & $\begin{array}{c}\text { Chinese } \\
\text { Pharmacopeia } \\
\text { (vision 2015) }\end{array}$ & - & $\leq 2.0 \%$ & - & $\begin{array}{c}\geq 0.12 \% \\
(\mathrm{ml} / \mathrm{g})\end{array}$ & - & $\begin{array}{l}\text { 6-Gingerol; } \\
\text { 8-Gingerol and } \\
\text { 10-gingerol }\end{array}$ & $\begin{array}{l}\quad \geq 0.05 \% ; \\
\text { The total } \\
\text { content } \\
\geq 0.04 \%\end{array}$ \\
\hline $\begin{array}{l}\text { Zingiberis } \\
\text { Rhizoma }\end{array}$ & $\begin{array}{l}\text { Dried } \\
\text { rhizome }\end{array}$ & $\begin{array}{c}\text { Chinese } \\
\text { Pharmacopeia } \\
\text { (vision 2015) }\end{array}$ & $\leq 19.0 \%$ & $\leq 6.0 \%$ & - & $\geq 0.8 \%(\mathrm{ml} / \mathrm{g})$ & $\geq 22.0 \%$ & 6-Gingerol & $\geq 0.6 \%$ \\
\hline $\begin{array}{l}\text { Zingiberis } \\
\text { Rhizoma }\end{array}$ & Rhizome & $\begin{array}{c}\text { Japanese } \\
\text { Pharmacopeia } \\
\text { (vision 2017) }\end{array}$ & - & $\leq 8.0 \%$ & - & - & - & 6-Gingerol & $\geq 0.3 \%$ \\
\hline $\begin{array}{l}\text { Zingiberis } \\
\text { Processum } \\
\text { Rhizoma }\end{array}$ & Rhizome & $\begin{array}{c}\text { Japanese } \\
\text { Pharmacopeia } \\
\text { (vision 2017) }\end{array}$ & $\leq 15.0 \%$ & $\leq 6.5 \%$ & $\leq 1.5 \%$ & - & $\geq 8.0 \%$ & - & - \\
\hline $\begin{array}{l}\text { Zingiberis } \\
\text { Rhizoma }\end{array}$ & $\begin{array}{l}\text { Dried } \\
\text { rhizome }\end{array}$ & $\begin{array}{l}\text { United States } \\
\text { Pharmacopeial } \\
\text { Convention } \\
\text { (vision 2017) }\end{array}$ & $\leq 10.0 \%$ & $\leq 8.0 \%$ & $\leq 2.0 \%$ & $\geq 1.8 \mathrm{ml} / 100 \mathrm{~g}$ & $\geq 10.0 \%$ & $\begin{array}{c}\text { Gingerols and } \\
\text { gingerdiones; } \\
\text { shogaols }\end{array}$ & $\begin{array}{l}\text { The total } \\
\text { content } \\
\geq 0.8 \% \leq \\
0.18 \%\end{array}$ \\
\hline $\begin{array}{l}\text { Zingiberis } \\
\text { Rhizoma }\end{array}$ & $\begin{array}{l}\text { Dried } \\
\text { rhizome }\end{array}$ & $\begin{array}{c}\text { European } \\
\text { pharmacopoeia } \\
\text { (vision } 8.0 \text { ) }\end{array}$ & $\begin{array}{c}\leq 100 \mathrm{ml} / \\
\mathrm{kg}\end{array}$ & $\leq 6.0 \%$ & - & $\geq 15 \mathrm{ml} / \mathrm{kg}$ & - & - & - \\
\hline $\begin{array}{l}\text { Zingiberis } \\
\text { Rhizoma }\end{array}$ & $\begin{array}{l}\text { Dried } \\
\text { rhizome }\end{array}$ & $\begin{array}{c}\text { Korean } \\
\text { Pharmacopoeia } \\
(\text { KP X) }\end{array}$ & - & $\leq 8.0 \%$ & - & - & - & 6-Gingerol & $\geq 0.4 \%$ \\
\hline Sunthi & $\begin{array}{l}\text { Dried } \\
\text { rhizome }\end{array}$ & $\begin{array}{c}\text { Indian } \\
\text { Pharmacopoeia } \\
\text { (vision 2010) }\end{array}$ & $\leq 12.0 \%$ & $\leq 8.0 \%$ & $\leq 1.5 \%$ & - & $\geq 2.0 \%$ & Total gingerols & $\geq 0.8 \%$ \\
\hline
\end{tabular}

Cited from the Drug Standard Database at https://www.drugfuture.com/standard/search.aspx. 
increasing doses of ZE (2, 4, 6, 8 and $10 \mathrm{~g} / \mathrm{kg})$ which were given to mice for 14 days, no toxic effects were observed compared with distilled water [89].

Although daily clinical administration of ZRR usually does not cause any significant adverse effects in humans, further clinical trials are required to better assess the safety of ZRR.

\section{Quality Control}

The quality of ZRR is affected by different factors such as growing environment and different producing areas. In addition, harvest time, processing methods, and storage conditions affect the quality of ZRR. The medicinal part of ZRR is the rhizoma, and it is often tangled whether to peel it. Due to these factors, the content of active ingredients in ZRR is different, and the quality and clinical efficacy of ZRR cannot be controlled at present.

In the current Pharmacopoeia of various countries, the quality control of $Z$. officinale is mainly carried out through qualitative identification by thin layer chromatography (TLC) and content is determined by high-performance liquid chromatography (HPLC). Table 7 shows the contents of $Z$. officinale in Pharmacopoeia of various countries.

In the Chinese Pharmacopoeia, TLC and microscopic observation are mainly used for qualitative identification and determination of content by HPLC, and gingerol detection is mainly used to evaluate the quality of ZRR. 6Ginerol, 8-gingerol, and 10-gingerol are chosen as three marker components to control the quality of ZRR, and it is required that the content of these marker components should be no less than the content specified in the method. ZRR and its processed products are often used in clinical practice.

TCM has characteristics of multicomponent and multitarget. Hence, it is insufficient to evaluate the quality of TCM in terms of the contents of a few compounds. Yet, chemical models of multicomponent control are increasingly common in the quality evaluations of TCM. To evaluate the quality of ZRR, some scholars have measured various components. For example, Zhang determined the contents of 6 kinds of gingerols (6-gingerol, 8-gingerol, 10gingerol, 6-shogaol, 8-shogaol, and 10-shogaol) and 4 kinds of curcumins (tetrahydrocurcumin, curcumin, demethoxycurcumin, and bis-demethoxycurcumin) [97]. Meng determined the content of gingerols by reverse highperformance liquid chromatography [98]. To evaluate the chemical constituents of ZRR more comprehensively, HPLC and UPLC fingerprinting was established for quality control of ZRR [99, 100]. In addition, fingerprinting was also used to analyze the quality of ZRR from different regions [100] and the composition and content changes during processing [101]. There are many volatile components in ZRR, and some scholars have established GC fingerprints of volatile components in ZRR and claimed that these methods can be used to evaluate the quality of ZRR $[102,103]$. Yet no quality assessment methods associated with the pharmacological activity of ZRR have been devised. It is obviously inadequate to evaluate the quality of ZRR based on chemical constituents alone, warranting the establishment of more activity-related or chemical-activity-based quality evaluation methods.

\section{Concluding and Perspectives}

ZRR is widely used as a medicine and food homologous variety, especially in tropical and subtropical regions. In traditional medicine, many prescriptions containing ZRR are still in use, such as Shengjiang Xiexin Tang and Guizhi Tang. Many clinical practices have proved that the application of ZRR in preventing vomiting is successful. Modern pharmacological research has gradually verified its antiemetic effect and discussed the anticancer, anti-inflammatory, and antioxidation effects. Extracts and isolates from ZRR have multiple medical functions, especially for the nervous system and the cardiovascular and cerebrovascular system. However, there are still some problems that need further study and discussion.

First of all, a variety of phytochemicals have been isolated from this plant. Gingerols are considered to be the main bioactive components, especially 6-gingerol. We have been reported many biological activities of 6-gingerol, and other components such as 6-shagaol have also been reported to have significant pharmacological activities, which deserves more attention.

Secondly, most pharmacological studies have only used a simple animal model, without further study of potential mechanisms of action, such as involved receptors or intercellular and intracellular pathways. In addition, the results of some methods used in animal research are not reliable due to the lack of the positive control group; in some studies, the lack of dose effect analysis has important limitations, and the application of dose needs further research to determine the optimal effective and safe dosage.

Thirdly, although ZRR possesses a variety of potential therapeutic effects, such as antioxidant, anti-inflammatory, neuroprotective, and cardio cerebrovascular protection, these studies have only been carried out in animal and cell models, and few clinical studies have been conducted in humans. As there is a long way to go from the test bed to the bedside, there is no acceptable evidence for clinical use of the plant. Future studies should focus on the biological activity of ZRR in human clinical studies. These studies will contribute to the future medical application of ZRR.

Fourth, the current quality control of ZRR is mainly based on the content requirements of gingerols. For example, 6-gingerol, 8-gingerol, and 10-gingerol are selected as the quality control indicators of ZRR in the Chinese Pharmacopoeia, and the minimum content is made. However, the content of ingredients cannot scientifically explain the quality of ZRR. The quality evaluation of ZRR should be related to clinical efficacy. The quality standard research of ZRR in the future is not only related to the content of components, but also to the pharmacological activity. In order to better define the quality of ZRR, the quality markers of pharmacodynamics are selected as the measurement indexes of quality standards. In recent years, the fingerprint analysis of 
traditional Chinese medicine is becoming more and more important in the analysis of traditional Chinese medicine. Future research can better define the content and change of components with the help of traditional Chinese medicine fingerprint, so as to ensure the stability of the quality of ZRR.

In conclusion, the botanical characterizations, traditional usages, chemical components, pharmacological activities, toxicity, and quality control of Zingiberis Rhizoma Recens were reviewed in this paper. In recent years, with the development of science and technology, ZRR has made significant progress in chemical components and pharmacological activities, but there are still some challenges. How to clarify the mechanism of traditional uses and modern pharmacological activities and how to establish the correlation research between chemical components and pharmacological activities are the focus of research. It is anticipated that this study can provide a basis for further study of ZRR and promote the long-term development.

\section{Abbreviations}

$\begin{array}{ll}\text { Z. officinale: } & \text { Zingiber officinale Roscoe } \\ \text { ZRR: } & \text { Zingiberis Rhizoma Recens } \\ \text { ZAE: } & \text { Aqueous extract of Zingiberis Rhizoma } \\ & \text { Recens } \\ \text { ZEE: } & \text { Ethanolic extract of Zingiberis Rhizoma } \\ & \text { Recens } \\ \text { ZSE: } & \text { Supercritical extract of Zingiberis Rhizoma } \\ & \text { Recens } \\ \text { ZME: } & \text { Methanol extract of Zingiberis Rhizoma } \\ & \text { Recens } \\ \text { AD: } & \text { Alzheimer's disease } \\ \text { CysLT1R: } & \text { Cysteinyl leukotriene 1 receptor } \\ \text { A } \beta: & \text { Amyloid beta } \\ \text { Akt: } & \text { Protein kinase } \\ \text { GSK-3 } \beta: & \text { Glycogen synthase kinase-3 } \beta \\ \text { PI3K: } & \text { Phosphoinositide-3 kinase } \\ \text { PC cells: } & \text { Pheochromocytoma cells } \\ \text { NGF: } & \text { Nerve growth factor } \\ \text { ERK: } & \text { Extracellular-signal-regulated kinase } \\ \text { CREB: } & \text { Cyclic AMP response element-binding } \\ & \text { protein } \\ \text { LPS: } & \text { Lipopolysaccharide } \\ \text { ROS: } & \text { Reactive oxygen species } \\ \text { BNIP3: } & \text { Bcl-2 E1B 19-KDa interacting protein } 3 \\ \text { TNF- } \alpha: & \text { Tumor necrosis factor- } \alpha \\ \text { CK: } & \text { Creatine kinase } \\ \text { LDH: } & \text { Lactate dehydrogenase } \\ \text { FCI-R: } & \text { Focal cerebral ischemia-reperfusion } \\ \text { MCAO: } & \text { Middle cerebral artery occlusion } \\ \text { Caspase: } & \text { Cysteinyl aspartate specific proteinase } \\ \text { Bax: } & \text { Bcl-2 associated X protein } \\ \text { Bcl-2: } & \text { B-cell lymphoma/leukemia-2 } \\ \text { CI-R: } & \text { Cerebral ischemia-reperfusion } \\ \text { EAA: } & \text { Excitatory amino acid } \\ \text { ICAM-1: } & \text { Intercellular adhesion molecule-1 } \\ \text { P-selectin: } & \text { Platelet-selectin } \\ \text { SOD: } & \text { Superoxide dismutase } \\ & \\ & \end{array}$

$\begin{array}{ll}\text { MDA: } & \text { Malondialdehyde } \\ \text { 5-HT: } & \text { 5-Hydroxytryptamine } \\ \text { NK: } & \text { Neurokinin } \\ \text { CINV: } & \text { Chemotherapy-induced nausea and vomiting } \\ \text { PONV: } & \text { Postoperative nausea and vomiting } \\ \text { MIC: } & \text { Minimum inhibitory concentration } \\ \text { STAT-3: } & \text { Signal transducer and activating } \\ & \text { transcription-3 } \\ \text { PARP-1: } & \text { Poly ADP ribose polymerase 1 } \\ \text { PGE2: } & \text { Prostaglandin E2 } \\ \text { NF- } \kappa \text { B: } & \text { Nuclear factor-kappa B } \\ \text { COX-2: } & \text { Cyclooxygenase-2 } \\ \text { INOs: } & \text { Inducible nitric oxide synthases } \\ \text { IL-1 } \beta: & \text { Interleukin-1 } \beta \\ \text { IL-6: } & \text { Interleukin-6 } \\ \text { IL-10: } & \text { Interleukin-10 } \\ \text { MAPK: } & \text { Mitogen-activated protein kinase } \\ \text { DAI: } & \text { Disease activity index } \\ \text { Nrf2: } & \text { Nuclear factor erythroid 2-related factor 2 } \\ \text { HO-1: } & \text { Hemeoxygenase-1 } \\ \text { DPPH: } & 1,1-\text { Diphenyl-2-picrylhydrazyl. } \\ \end{array}$

\section{Additional Points}

We followed the methods of Hanyan Luo et al., 2019 [104].

\section{Disclosure}

Xing Li and Mingyue Ao are regarded as co-first authors.

\section{Conflicts of Interest}

The authors declare that there are no conflicts of interest.

\section{Authors' Contributions}

Xing Li and Mingyue Ao contributed equally to this work. Chunling Zhang helped checking the chemical structures and formula. Shunming Fan downloaded the documents and made classification. Zhimin Chen checked the manuscript and pointed out the mistakes. Lingying Yu approved the final version of the manuscript. Xing Li and Mingyue Ao contributed to the work equally.

\section{Acknowledgments}

This work was financially supported by the Open Research Fund of Chengdu University of Traditional Chinese Medicine Key Laboratory of Systematic Research of Distinctive Chinese Medicine Resources in Southwest China (2020JCRC022).

\section{References}

[1] Institute of Botany and the Chinese Academy of Sciences, Flora of China, Science Press, Beijing, China, 1992.

[2] Z. Q. Lei and T. M. Zhang, Chinese Clinical Chinese Medicine, People's Medical Publishing House, Beijing, China, 1998. 
[3] X. Y. Sun and F. Y. Sun, Shennong Ben Cao Jing, Shanxi Publishing Media Group, Taiyuan, China, 2010.

[4] Z. J. Zhang, Jin Gui Yao Lue, People's Medical Publishing House, Beijing, China, 2005.

[5] Z. J. Zhang, Shang Han Lun, Shanghai Science and Technology Press, Shanghai, China, 2018.

[6] J. Li, Z. M. Wang, and H. M. Gao, "The effect of the process on the volatile components in the fresh ginger and its processed products," Chinese Journal of Experimental Traditional Medical Formulae, vol. 18, no. 19, pp. 77-81, 2012.

[7] Y. P. Yang, J. Du, X. M. Deng, and J. Meng, "Content determination of gingerols in ginger and its different processed products," Journal of Modern Medicine and Health, vol. 31, no. 22, pp. 3369-3370, 2015.

[8] S. X. Zhao and J. Mao, "Determination of content of polysaccharide in fresh ginger and its processed products," China Journal of Chinese Medicine, vol. 26, no. 12, pp. 1475-1476, 2011.

[9] S. Z. Jiang, S. Q. Mi, and N. S. Wang, "Review of the chemical constituents of gingerols," Traditional Chinese Drug Research and Clinical Pharmacology, vol. 5, pp. 386-389, 2006.

[10] H. Xiong, The Compare Research of Ingredient of Ginger Extraction with Different Distilling Methods, Xihua University, Chengdu, China, 2006.

[11] T. C. Li, D. Y. Hou, R. H. Hui, and Q. P. Diao, "Analysis of volatile components of ginger and galangal," Journal of Anshan Normal University, vol. 20, no. 6, pp. 38-40, 2018.

[12] C. S. Huang, S. P. Bai, and Y. Li, "Natural linear diarylheptanes," Natural Product Research and Development, vol. 2, pp. 98-105, 1997.

[13] S. B. Tan, Study on the Water-Soluble Chemical Constituents of Rhizome and Acrial Part of Zingiber Officinale Rosc, Lanzhou University of Technology, Lanzhou, China, 2017.

[14] R. P. Zhang, J. L. Hu, Y. Zhao, X. D. Zhang, and G. Liu, "Analysis of amino acids and trace elements in fresh ginger," Journal of Traditional Chinese Medicine Research, vol. 1, p. 44, 2002.

[15] L. Y. Zhong, H. L. Tong, J. Zhu, and M. Lv, "Pharmacological effects of different ginger juices on the concurrent symptoms in animal models of functional dyspepsia: a comparative study," Food Science \& Nutrition, vol. 7, no. 7, pp. 2205-2213, 2019.

[16] Z. B. Wang, G. F. Xin, X. Y. Song, S. S. Jia, and K. Xu, "Processing quality and oleoresin composition of ginger at different growth periods," Food Science, vol. 34, no. 6, pp. 6-9, 2013.

[17] Y. Li, Y. Hong, Y. Han, Y. Wang, and L. Xia, "Chemical characterization and antioxidant activities comparison in fresh, dried, stir-frying and carbonized ginger," Journal of Chromatography B, vol. 1011, pp. 223-232, 2016.

[18] V. P. Baldin, R. Bertin de Lima Scodro, C. M. Mariano Fernandez et al., "Ginger essential oil and fractions against Mycobacterium spp," Journal of Ethnopharmacology, vol. 15, p. 224, 2019.

[19] J. J. Cui and Q. Li, "Comparative analysis of volatile components of dfferent ginger extractionsu," China Food Additives, vol. 2, pp. 55-61, 2010.

[20] L. X. Wang, W. H. Zhao, Y. F. Lu, and C. X. Wang, "Antioxidant and cytotoxic activities of distillates purified by means of molecular distillation from ginger extract obtained with supercritical $\mathrm{CO}_{2}$ fluid," Chemistry and Biodiversity, vol. 16, no. 11, 2019.
[21] K. Y. Zhan, Analysis of the Chemical Composition of Ginger Oil and the Separation and Purification of Gingerol, Shandong Agricultural University, Tai'an, China, 2009.

[22] J. Ma, X. L. Jin, L. Yang, and Z. L. Liu, "Diarylheptanoids from the rhizomes of Zingiber officinale," Phytochemistry, vol. 65, no. 8, pp. 1137-1143, 2004.

[23] L. X. Yang, C. X. Zhou, K. X. Huang et al., "Antioxidative and cytotoxic properties of diarylheptanoids isolated from Zingiber officinale," China Journal of Chinese Materia Medica, vol. 34, no. 3, pp. 319-323, 2009.

[24] H. Kikuzaki and N. Nakatani, "Cyclic diarylheptanoids from rhizomes of Zingiber officinale," Phytochemistry, vol. 43, no. 1, pp. 273-277, 1996.

[25] G. F. Zeng, S. H. Zong, J. M. He, Z. Y. Zhang, L. Lv, and D. Q. Xiao, "Effect and its mechanism of ginger root extract on cognitive dysfunction in rats with Alzheimer disease," Journal of Clinical Neurology, vol. 26, no. 5, pp. 362-365, 2013.

[26] J.-Y. Na, K. Song, J.-W. Lee, S. Kim, and J. Kwon, "6-Shogaol has anti-amyloidogenic activity and ameliorates Alzheimer's disease via CysLT1R-mediated inhibition of cathepsin B," Biochemical and Biophysical Research Communications, vol. 477, no. 1, pp. 96-102, 2016.

[27] G. F. Zeng, S. H. Zong, S. W. Fu, M. N. Nong, K. K. Li, and F. N. Yang, "Protective effect of 6-gingerol on PC12 Cells induced by Amyloid $\beta$-Protein by PI3K/Akt/GSK-3 $\beta$ signaling pathway," Natural Product Research and Development, vol. 27, no. 11, pp. 1862-1865, 2015.

[28] E. Huh, S. Lim, H. G. Kim et al., "Ginger fermented with Schizosaccharomyces pombe alleviates memory impairment via protecting hippocampal neuronal cells in amyloid beta142 plaque injected mice," Food \& Function, vol. 9, no. 1, pp. 171-178, 2018.

[29] Z. C. Kang, Y. R. Li, and M. Z. Ao, "Effect of ginger on nitric oxide content and learning and memory in hippocampus of lead exposed rats," Chinese Journal of Gerontology, vol. 38, no. 4, pp. 940-942, 2018.

[30] S. Lim, M. Moon, H. Oh, H. G. Kim, S. Y. Kim, and M. S. Oh, "Ginger improves cognitive function via NGF-induced ERK/ CREB activation in the hippocampus of the mouse," The Journal of Nutritional Biochemistry, vol. 25, no. 10, pp. 1058-1065, 2014.

[31] F. Zhang, J.-G. Zhang, W. Yang, P. Xu, Y.-L. Xiao, and H.-T. Zhang, "6-Gingerol attenuates LPS-induced neuroinflammation and cognitive impairment partially via suppressing astrocyte overactivation," Biomedicine \& Pharmacotherapy, vol. 107, pp. 1523-1529, 2018.

[32] A. A. El-Hawwary and N. M. Omar, "The influence of ginger administration on cisplatin-induced cardiotoxicity in rat: light and electron microscopic study," Acta Histochemica, vol. 121, no. 5, pp. 553-562, 2019.

[33] X. Han, Myocardial Protective Effect and Mechanism of 6gingerol Based on TLR4/MAPKs/NF- $\kappa B$ Signaling Pathway and Calcium Channel, Hebei University of Chinese Medicine, Hebei, China, 2019.

[34] X. W. Lv and T. T. Xu, "6-gingerol inhibits oxidative stress ameliorated myocardial ischemia/reperfusion injury in rats," Journal of Clinical Cardiology, vol. 33, no. 6, pp. 575-579, 2017.

[35] W. Zhang, X. Liu, Y. Jiang, N. Wang, F. Li, and H. Xin, "6gingerol attenuates ischemia-reperfusion-induced cell apoptosis in human AC16 cardiomyocytes through HMGB2JNK1/2-NF- $\kappa B$ pathway," Evidence-Based Complementary and Alternative Medicine, vol. 2019, pp. 1-8, 2019. 
[36] X. Han, Y. Zhang, Y. Liang et al., "6-gingerol, an active pungent component of ginger, inhibits L-type $\mathrm{Ca}^{2+}$ current, contractility, and $\mathrm{Ca}^{2+}$ transients in isolated rat ventricular myocytes," Food Science \& Nutrition, vol. 7, no. 4, pp. 1344-1352, 2019.

[37] T. Xu, G. Qin, W. Jiang, Y. Zhao, Y. Xu, and X. Lv, "Corrigendum to "6-gingerol protects heart by suppressing myocardial ischemia/reperfusion induced inflammation via the PI3K/Akt-Dependent mechanism in rats"' EvidenceBased Complementary and Alternative Medicine, vol. 2019, pp. 1-2, 2019.

[38] Q. Ren, S. Zhao, and C. Ren, "6-gingerol protects cardiocytes H9c2 against hypoxia-induced injury by suppressing BNIP3 expression," Artificial Cells, Nanomedicine, and Biotechnology, vol. 47, no. 1, pp. 2016-2023, 2019.

[39] P. Tian, D. W. Zhang, K. Ma, J. Wang, and Y. Cui, "Effect of ginger total phenol on focal cerebral ischemic model in rats," Pharmacology and Clinics of Chinese Materia Medica, vol. 33, no. 5, pp. 70-73, 2017.

[40] S. Q. Jia, J. Wang, H. X. Zhang, X. Huang, W. Sun, and M. H. Fu, "Effects of zingiberis rhizoma recens on neuronal apoptosis and related prote in expression in focal cerebral ischemia-reperfusion in rats," Chinese Journal of Experimental Traditional Medical Formulae, vol. 17, no. 3, pp. 163-166, 2011.

[41] J. Wang, Q. F. Huang, H. X. Liu, and W. Zhang, "Effect of aqueous extract from zingiberis rhizoma recens on excitatory amino acid in brain tissue of cerebral ischemia reperfusion in rats in vivo," Chinese Journal of Experimental Traditional Medical Formulae, vol. 17, no. 21, pp. 184-187, 2011.

[42] J. Wang, H. X. Zhang, S. Q. Jia, and Y. S. Wang, "Effects of aqueous extract from ginger on inflammatory cytokine and adhesion molecule in brain tissue of cerebral ischemia reperfusion in rats in vivo," Journal of Medical Forum, vol. 32, no. 15, pp. 12-14, 2011.

[43] G. T. Zhang, J. Wang, L. Zhang, H. X. Liu, and W. Zhang, "Effect of ginger aqueous extract on coagulation function in rats with global cerebral ischemia-reperfusion," Traditional Chinese Medicinal Research, vol. 4, pp. 18-20, 2007.

[44] L. L. Song, J. Q. Sha, L. Zhang, and Y. X. Zhang, "Study on extraction of ginger polysaccharide and its protecting effect on cerebral ischemia- reperfusion injury," Liaoning Journal of Traditional Chinese Medicine, vol. 42, no. 15, pp. 24332435, 2015.

[45] X. X. Hu, X. Liu, Y. Chu, W. X. Chen, K. W. Zhang, and $\mathrm{H}$. Wu, "Antiemetic activity of effective extract and bioactive compounds in ginger," China Journal of Chinese Materia Medica, vol. 41, no. 5, pp. 904-909, 2016.

[46] J. Du, Q. L. Zhang, G. S. Li, and K. Nie, "Effects of ginger and its pungent constituents on gastrointestinal tract:role of $\mathrm{NK}_{1}$ receptors in isolated ileum of Guinea pig," Journal of Shandong University of Traditional Chinese Medicine, vol. 41, no. 4, pp. 364-367, 2017.

[47] J. Walstab, D. Krüger, T. Stark et al., "Ginger and its pungent constituents non-competitively inhibit activation of human recombinant and native $5-\mathrm{HT}_{3}$ receptors of enteric neurons," Neurogastroenterology \& Motility, vol. 25, no. 5, pp. 439e302, 2013

[48] L. Y. Zhang, "Antiemetic effect of ginger acetone extract on rat model of heterophilic kaolin," Guide of China Medicine, vol. 9, pp. 23-26, 2008.

[49] W. J. Liu, Y. Cui, B. Ji, J. M. Wang, and Z. Y. Feng, "Study on the material foundation of ginger antiemetic efficacy," China Journal of Chinese Medicine, vol. 28, no. 3, pp. 388-389, 2013.
[50] J. Du, Q. L. Zhang, G. S. Li, and K. Nie, "Effects of rhizoma zingiberis recens on $5-\mathrm{HT}_{3}$ receptor mediated contractile activity of isolated Guinea-pig ileum," Traditional Chinese Drug Research \& Clinical Pharmacolog, vol. 27, no. 5, pp. 632-636, 2016.

[51] F. Sharifzadeh, M. Kashanian, J. Koohpayehzadeh, F. Rezaian, N. Sheikhansari, and N. Eshraghi, "A comparison between the effects of ginger, pyridoxine (vitamin B6) and placebo for the treatment of the first trimester nausea and vomiting of pregnancy (NVP)," The Journal of MaternalFetal \& Neonatal Medicine, vol. 31, no. 19, pp. 2509-2514, 2018.

[52] A. Uthaipaisanwong, S. Oranratanaphan, and N. Musigavong, "Effects of ginger adjunct to the standard prophylaxis on reducing carboplatin and paclitaxel-induced nausea vomiting: a randomized controlled study," Supportive Care in Cancer, vol. 28, no. 8, pp. 3831-3838, 2020.

[53] W. P. Chang and Y. X. Peng, "Does the oral administration of ginger reduce chemotherapy-induced nausea and vomiting?: a meta-analysis of 10 randomized controlled trials," Cancer Nursing, vol. 42, no. 6, pp. 14-23, 2019.

[54] J. Konmun, K. Danwilai, N. Ngamphaiboon, B. Sripanidkulchai, A. Sookprasert, and S. Subongkot, "A phase II randomized double-blind placebo-controlled study of 6-gingerol as an anti-emetic in solid tumor patients receiving moderately to highly emetogenic chemotherapy," Medical Oncology, vol. 34, no. 4, p. 69, 2017.

[55] E. Soltani, A. Jangjoo, M. Afzal Aghaei, and A. Dalili, "Effects of preoperative administration of ginger (Zingiber officinale Roscoe) on postoperative nausea and vomiting after laparoscopic cholecystectomy," Journal of Traditional and Complementary Medicine, vol. 8, no. 3, pp. 387-390, 2017.

[56] L. X. Wang, J. Qian, S. Feng, J. L. Zhang, H. H. Hou, and S. Y. Wang, "Antioxidant and antimicrobial activities of volatile oil from ginger by supercritical extraction," Food Research and Development, vol. 37, no. 13, pp. 31-34, 2016.

[57] M. Aghazadeh, A. Zahedi Bialvaei, M. Aghazadeh et al., "Survey of the antibiofilm and antimicrobial effects of Zingiber officinale (in vitro study)," Jundishapur Journal of Microbiology, vol. 9, no. 2, 2016.

[58] A. A. Yusuf, B. Lawal, A. N. Abubakar et al., "In-vitro antioxidants, antimicrobial and toxicological evaluation of Nigerian Zingiber officinale," Clinical Phytoscience, vol. 4, no. 1, p. 12, 2018.

[59] D. Liu and J. P. Yan, "In vitro antibacterial effect of ginger extract," Studies of Trace Elements and Health, vol. 27, no. 2, pp. 33-34, 2010.

[60] J. R. Han and D. H. Yang, "Study on antibacterial effect of crude ginger extract," Journal of Community Medicine, vol. 12, no. 18, pp. 81-82, 2014.

[61] L. Song, Y. He, X. B. Zhang, and R. J. Yuan, "Extraction and antibacterial effect of polyphenols in ginger," Journal of Xinyang Normal University (Natural Science Edition), vol. 30, no. 3, pp. 445-448, 2017.

[62] C. X. Guo, Z. X. Wen, Z. H. Liu, and Y. J. Xu, "Enzyme assisted extraction and antimicrobial activity analysis of flavonoids from ginger," Journal of Yibin University, vol. 20, no. 6, pp. 99-103, 2020.

[63] P. Li, Z. Shu, C. Y. Hu, J. K. Yan, and Q. Q. Wang, "Ultrasonic extraction of gingerol and its antibacterial activity," China Condiment, vol. 42, no. 10, pp. 160-164, 2017.

[64] D. P. Mansingh, S. Pradhan, D. Biswas, R. Barathidasan, and H. R. Vasanthi, "Palliative role of aqueous ginger extract on 
N-nitroso-N-methylurea-induced gastric cancer," Nutrition and Cancer, vol. 72, no. 1, pp. 157-169, 2020.

[65] Q. Z. Wang, "Effects of Zingiber officinale extraction in alcohol on mouse testis sertoli cell line $\mathrm{TM}_{4}$," Journal of Weifang University, vol. 12, no. 4, pp. 60-63, 2012.

[66] A. I. Elkady, O. A. Abu-Zinadah, and R. A. E. H. Hussein, "Crude flavonoid extract of medicinal herb Zingibar officinale inhibits proliferation and induces apoptosis in hepatocellular carcinoma cells," Oncology Research Featuring Preclinical and Clinical Cancer Therapeutics, vol. 25, no. 6, pp. 897-912, 2017.

[67] S. Xu, H. Zhang, T. Liu et al., "6-gingerol induces cell-cycle G1-phase arrest through AKT-GSK $3 \beta$-cyclin D1 pathway in renal-cell carcinoma," Cancer Chemotherapy and Pharmacology, vol. 85, no. 2, pp. 379-390, 2020.

[68] V. Kapoor, S. Aggarwal, and S. N. Das, "6-gingerol mediates its anti tumor activities in human oral and cervical cancer cell lines through apoptosis and cell cycle arrest," Phytotherapy Research, vol. 30, no. 4, pp. 588-595, 2016.

[69] C. B. Lin, C. C. Lin, and G. J. Tsay, "6-gingerol inhibits growth of colon cancer cell LoVo via induction of G2/M arrest," Evidence-Based Complementary and Alternative Medicine, vol. 2012, no. 6, Article ID 326096, 2012.

[70] J. B. Yu and M. J. Shao, "The effect and mechanism of 6gingerol on the proliferation and apoptosis of human breast cancer cells," Chinese Journal of Gerontology, vol. 39, no. 16, pp. 4072-4075, 2019.

[71] S. Wang, X. Sun, L. Jiang et al., "6-gingerol induces autophagy to protect HUVECs survival from apoptosis," Chemico-Biological Interactions, vol. 256, pp. 249-256, 2016.

[72] J. Fan, X. Yang, and Z. Bi, "6-gingerol inhibits osteosarcoma cell proliferation through apoptosis and AMPK activation," Tumor Biology, vol. 36, no. 2, pp. 1135-1141, 2015.

[73] D. P. Mansingh, O. J. Sunanda, V. K. Sali, and H. R. Vasanthi, "[6]-gingerol-induced cell cycle arrest, reactive oxygen species generation, and disruption of mitochondrial membrane potential are associated with apoptosis in human gastric cancer (AGS) cells," Journal of Biochemical and Molecular Toxicology, vol. 32, no. 10, 2018.

[74] I.-J. Yeh, S.-C. Chen, M.-C. Yen, Y.-H. Wu, C.-H. Hung, and P.-L. Kuo, "6-shogaol suppresses 2-amino-1-methyl-6-phenylimidazo [4, 5-b] pyridine (PhIP)-induced human 786-O renal cell carcinoma osteoclastogenic activity and metastatic potential," Nutrients, vol. 11, no. 10, p. 2306, 2019.

[75] T. Liang, Y. He, Y. Chang, and X. Liu, "6-shogaol a active component from ginger inhibits cell proliferation and induces apoptosis through inhibition of STAT-3 translocation in ovarian cancer cell lines (A2780)," Biotechnology and Bioprocess Engineering, vol. 24, no. 3, pp. 560-567, 2019.

[76] U. Kotowski, L. Kadletz, S. Schneider et al., "6-shogaol induces apoptosis and enhances radiosensitivity in head and neck squamous cell carcinoma cell lines," Phytotherapy Research, vol. 32, no. 2, pp. 340-347, 2018.

[77] P. Su, V. P. Veeraraghavan, S. Krishna Mohan, and W. Lu, “A ginger derivative, zingerone-a phenolic compound-induces ROS-mediated apoptosis in colon cancer cells (HCT-116)," Journal of Biochemical and Molecular Toxicology, vol. 33, no. 12,2019

[78] H. Y. Gan, Y. Q. Zhang, Q. Y. Zhou, L. R. Zheng, X. F. Xie, and V. P. Veeraraghavan, "Zingerone induced caspase-dependent apoptosis in MCF-7 cells and prevents 7, 12dimethylbenz (a) anthracene-induced mammary carcinogenesis in experimental rats," Journal of Biochemical and Molecular Toxicology, vol. 33, no. 10, 2019.
[79] J.-S. Choi, J. Ryu, W.-Y. Bae et al., "Zingerone suppresses tumor development through decreasing cyclin D1 expression and inducing mitotic arrest," International Journal of Molecular Sciences, vol. 19, no. 9, p. 2832, 2018.

[80] M. S. Kim and J. Y. Kim, "Ginger attenuates inflammation in a mouse model of dextran sulfate sodium-induced colitis," Food Science and Biotechnology, vol. 27, no. 5, pp. 1493-1501, 2018.

[81] S. Murugesan, M. R. Venkateswaran, S. Jayabal, and S. Periyasamy, "Evaluation of the antioxidant and antiarthritic potential of Zingiber officinale Rosc. by in vitro and in silico analysis," South African Journal of Botany, vol. 130, pp. 45-53, 2020.

[82] S. M. Ezzat, M. I. Ezzat, M. M. Okba, E. T. Menze, and A. B. Abdel-Naim, "The hidden mechanism beyond ginger (Zingiber officinale Rosc.) potent in vivo and in vitro antiinflammatory activity," Journal of Ethnopharmacology, vol. 214, pp. 113-123, 2018.

[83] G. T. Yocum, J. J. Hwang, M. Mikami, J. Danielsson, A. S. Kuforiji, and C. W. Emala, "Ginger and its bioactive component 6-shogaol mitigate lung inflammation in a murine asthma model," American Journal of Physiology-Lung Cellular and Molecular Physiology, vol. 318, no. 2, pp. 296303, 2020.

[84] B. Ajayi, I. Adedara, and E. Farombi, "Protective mechanisms of 6-gingerol in dextran sulfate sodium-induced chronic ulcerative colitis in mice," Human \& Experimental Toxicology, vol. 37, no. 10, pp. 1054-1068, 2018.

[85] K.-W. Chang and C.-Y. Kuo, "6-Gingerol modulates proinflammatory responses in dextran sodium sulfate (DSS)treated Caco-2 cells and experimental colitis in mice through adenosine monophosphate-activated protein kinase (AMPK) activation," Food \& Function, vol. 6, no. 10, pp. 3334-3341, 2015.

[86] F. Chen, Y. Tang, Y. Sun, V. P. Veeraraghavan, S. K. Mohan, and C. Cui, "6-shogaol, a active constiuents of ginger prevents UVB radiation mediated inflammation and oxidative stress through modulating NrF2 signaling in human epidermal keratinocytes (HaCaT cells)," Journal of Photochemistry and Photobiology B: Biology, vol. 197, Article ID 111518, 2019.

[87] Y. G. Kim, M. O. Kim, S. H. Kim et al., "6-shogaol, an active ingredient of ginger, inhibits osteoclastogenesis and alveolar bone resorption in ligature-induced periodontitis in mice," Journal of Periodontology, vol. 91, no. 6, pp. 809-818, 2020.

[88] Y.-H. Hwang, T. Kim, R. Kim, and H. Ha, "The natural product 6-gingerol inhibits inflammation-associated osteoclast differentiation via reduction of prostaglandin E2 Levels," International Journal of Molecular Sciences, vol. 19, no. 7, p. 2068, 2018.

[89] O. Bekkouch, M. Harnafi, I. Touiss et al., "In vitro antioxidant and in vivo lipid-lowering properties of Zingiber officinale crude aqueous extract and methanolic fraction: a follow-up study," Evidence-Based Complementary and Alternative Medicine, vol. 2019, Article ID 9734390, 2019.

[90] E. T. Mohammed, K. S. Hashem, A. E. Ahmed, M. T. Aly, L. Aleya, and M. M. Abdel-Daim, "Ginger extract ameliorates Bisphenol A (BPA)-induced disruption in thyroid hormones synthesis and metabolism: involvement of Nrf-2/ HO-1 pathway," Science of The Total Environment, vol. 703, Article ID 134664, 2020.

[91] A. Hosseinzadeh, K. Bahrampour Juybari, M. J. Fatemi et al., "Protective effect of ginger (zingiber officinale Roscoe) extract against oxidative stress and mitochondrial apoptosis 
induced by interleukin- $1 \beta$ in cultured chondrocytes," Cells Tissues Organs, vol. 204, no. 5-6, pp. 241-250, 2017.

[92] K. Ji, L. Fang, H. Zhao et al., "Ginger oleoresin alleviated $\gamma$-ray irradiation-induced reactive oxygen species via the Nrf2 protective response in human mesenchymal stem cells," Oxidative Medicine and Cellular Longevity, vol. 2017, 12 pages, 2017.

[93] F. Wang, Y. A. Huang, X. Shen, and J. Lu, "The test of acute toxicity on different ginger extract," Food Research and Development, vol. 30, no. 1, pp. 138-140, 2009.

[94] T. Plengsuriyakarn and K. Na-Bangchang, "Preclinical toxicology and anticholangiocarcinoma activity of oral formulation of standardized extract of Zingiber officinale," Planta Medica, vol. 86, no. 2, pp. 104-112, 2020.

[95] M. A. Okesola, B. O. Ajiboye, B. E. Oyinloye, and O. A. Ojo, "Effect of Zingiber officinaleon some biochemical parameters and cytogenic analysis in lead-induced toxicity in experimental rats," Toxicology Mechanisms and Methods, vol. 29, no. 4, pp. 255-262, 2019.

[96] A. Akbari, K. Nasiri, M. Heydari, S. H. Mosavat, and A. Iraji, "The protective effect of hydroalcoholic extract of Zingiber officinale Roscoe (Ginger) on ethanol-induced reproductive toxicity in male rats," Journal of Evidence-Based Complementary \& Alternative Medicine, vol. 22, no. 4, pp. 609-617, 2017.

[97] W. H. Zhang, Comparative Analysis of Ginger Quality Safety Standards and Different Study on Characteristic Components, Chinese Academy of Agricultural Sciences, Beijing, China, 2019.

[98] X. C. Meng, "Content determination of 3 gingerols in dried ginger, baked ginger and fresh ginger by RP-HPLC," China Pharmacy, vol. 22, no. 23, pp. 2182-2184, 2011.

[99] K. Ma, P. Tian, D. W. Zhang, and Y. Cui, "The optimization study of the HPLC-DAD fingerprint for Zingiber officinale," Lishizhen Medicine and Materia Medica Research, vol. 28, no. 9, pp. 2051-2054, 2017.

[100] Y. Q. Han, Y. Hong, J. Gui, J. R. Gao, Y. Z. Wang, and L. Z. Xia, "UPLC fingerprint comparison among fresh, dried and processed ginger from different habitats," Chinese Traditional Patent Medicine, vol. 35, no. 2, pp. 356-359, 2013.

[101] W. H. Wang, J. Li, H. M. Gao, Z. M. Wang, and L. Zhang, "Chemical ingredients change of ginger processing with HPLC specific chromatogram," Chinese Journal of Pharmaceutical Analysis, vol. 29, no. 8, pp. 1248-1252, 2009.

[102] T. L. Huang, X. Hong, and N. S. Wang, "Research on gas chromatographic fingerprint of ginger volatile oil components," Traditional Chinese Drug Research and Clinical Pharmacology, vol. 2, pp. 123-124, 2003.

[103] Q. Yang and G. D. Zhang, "Determination of volatile compounds in ginger by gas chromatography-mass spectrometry," China Condiment, vol. 44, no. 7, pp. 129-132, 2019.

[104] H. Luo, H. Wu, X. Yu et al., "A review of the phytochemistry and pharmacological activities of magnoliae officinalis cortex," Journal of Ethnopharmacology, vol. 236, no. 236, pp. 412-442, 2019. 